\title{
On the detectability of Ly $\alpha$ emission in star forming galaxies
}

\section{The role of dust $\star, \star \star, \star \star \star$}

\author{
H. Atek ${ }^{1}$, D. Kunth ${ }^{1}$, M. Hayes ${ }^{2, \dagger}$, G. Östlin ${ }^{2}$, and J. M. Mas-Hesse ${ }^{3}$ \\ 1 Institut d'Astrophysique de Paris (IAP), 98bis boulevard Arago, 75014 Paris, France \\ e-mail: atek@iap.fr \\ 2 Stockholm Observatory, AlbaNova University Centre, 10691 Stockholm, Sweden \\ 3 Centro de Astrobiología (CSIC-INTA), 28850 Torrejón de Ardoz, Spain \\ Received 6 February 2008 / Accepted 20 May 2008
}

ABSTRACT

\begin{abstract}
Context. Lyman-alpha ( $\operatorname{Ly} \alpha)$ radiation is now widely used to investigate the galaxy formation and evolution in the high redshift universe. However, without a rigorous understanding of the processes regulating the Ly $\alpha$ escape fraction, physical interpretations of high- $z$ observations remain questionable.

Aims. We examine six nearby star forming galaxies to disentangle the role of the dust from other parameters such as gas kinematics, geometry, and ISM morphology in the obscuration of Ly $\alpha$. Thereby, we attempt to understand the Ly $\alpha$ escape physics and infer the implications for high-redshift studies.

Methods. We use HST/ACS imaging to produce continuum-subtracted Ly $\alpha$ maps, and ground-based observations (ESO/NTT and NOT) to map the $\mathrm{H} \alpha$ emission and the extinction $E(B-V)$ in the gas phase derived from the Balmer decrement $\mathrm{H} \alpha / \mathrm{H} \beta$.

Results. When large outflows are present, the Ly $\alpha$ emission does not appear to be correlated with the dust content, confirming the role of the HI kinematics in the escape of Ly $\alpha$ photons. In the case of a dense, static HI covering, we observe a damped absorption with a negative correlation between $\operatorname{Ly} \alpha$ and $E(B-V)$. We found that the Ly $\alpha$ escape fraction does not exceed $10 \%$ in all our galaxies and is mostly about 3\% or below. Finally, because of the radiative transfer complexity of the Ly $\alpha$ line, star formation rate based on Ly $\alpha$ luminosity is underestimated with respect to that derived from UV luminosity. Simple reddening correction does not reconcile $\operatorname{SFR}(\operatorname{Ly} \alpha)$ with the total star formation rate.

Conclusions. The dust is not necessarily the main Ly $\alpha$ escape regulatory factor. ISM kinematics and geometry may play a more significant role. The failure of simple dust correction to recover the intrinsic $\mathrm{Ly} \alpha / \mathrm{H} \alpha$ ratio or the total star formation rate should prompt us to be more cautious when interpreting high- $z$ observations and related properties, such as SFRs based on Ly $\alpha$ alone. To this end, we propose a more realistic calibration for $\operatorname{SFR}(\operatorname{Ly} \alpha)$, which accounts for dust attenuation and resonant scattering effects via the Ly $\alpha$ escape fraction.
\end{abstract}

Key words. galaxies: starburst - galaxies: ISM - ultraviolet: galaxies - ISM: dust, extinction - galaxies: general

\section{Introduction}

The Lyman-alpha emission $(\operatorname{Ly} \alpha)$ has become the most powerful tracer of star formation in the high redshift universe. It is the strongest emission line at optical and near infrared (NIR) wavelengths at redshift $z \geq 2.1$, and is likely to remain a competitive tool, even with the advent of extremely large telescopes (ELTs) and the James Webb Space Telescope (JWST). It is widely used as an efficient detection and redshift confirmation tool for distant galaxies, to derive star formation rates (SFRs), as well as

* This work was supported by the Centre National d'Études Spatiales (CNES). It is based on observations made with the NASA/ESA Hubble Space Telescope, obtained at the Space Telescope Science Institute, which is operated by the Association of Universities for Research in Astronomy, Incorporated under NASA contract NAS 5-26555. These observations are associated with programs \#GO 9470 and \#GO 10575.

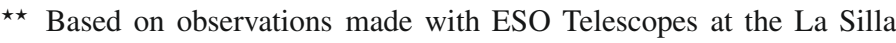
Observatories under programme IDs 073.B-0785 and 70.B-0639.

$\star \star \star$ A part of data presented here have been taken using ALFOSC, which is owned by the Instituto de Astrofisica de Andalucia (IAA) and operated at the Nordic Optical Telescope under agreement between IAA and the NBIfAFG of the Astronomical Observatory of Copenhagen.

$\dagger$ Present address: observatoire de Genève, 51, Ch. des Maillettes, 1290, Sauverny, Switzerland. to probe the ionization state of the intergalactic medium (IGM) at the final stage of the reionization epoch. In this way, the past decade has been the high-redshift era, in which the development of new techniques and facilities have enabled galaxy physical properties to be measured directly, providing a major improvement in our understanding of the distant Universe.

The importance of the Ly $\alpha$ emission line in the cosmological context was predicted initially by Partridge \& Peebles (1967), who suggested that young high- $z$ galaxies, undergoing their first star formation events, should be detectable because of their strong Ly $\alpha$ emission. Unfortunately, the first attempts to detect such objects contradicted those predictions. Initial surveys (e.g. Pritchet \& Hartwick 1989; Djorgovski \& Thompson 1992; de Propris et al. 1993) failed to discover the predicted space density of Ly $\alpha$ emitters. These unsuccessful campaigns and the faint measured Ly $\alpha$ fluxes were attributed to the dust attenuation coupled to the resonant scattering of the Ly $\alpha$ line (see Pritchet 1994, for a review). The first breakthrough was achieved by the observations of Cowie \& Hu (1998) and $\mathrm{Hu}$ et al. (1998), which led to the development of high-efficiency surveys for high-z LAEs detection. Two techniques are now used routinely to detect high- $z$ galaxies. Firstly, the Lyman Break Technique (Steidel et al. 1996) uses the absorption bluewards of the Ly $\alpha$ absorption edge to detect the so-called Lyman Break Galaxies (LBGs). 
Secondly, narrow-band imaging surveys use the Ly $\alpha$ recombination line emission produced by the reprocessed absorbed radiation to target Lyman-Alpha Emitters (LAEs). These successful techniques now provide a central role in our attempts to understand the distant universe. Besides its use in the identification of galaxies, it is used to place constraints on the cosmic reionization (Malhotra \& Rhoads 2004; Kashikawa et al. 2006; Dijkstra et al. 2007), and to study the clustering properties and morphology of galaxies to the highest redshifts (Hamana et al. 2004; Ouchi et al. 2005; Murayama et al. 2007). In addition, it enables star formation rates to be measured to high redshift (Kudritzki et al. 2000; Fujita et al. 2003; Pirzkal et al. 2007).

The Ly $\alpha$ star formation rate is derived typically by applying the $\mathrm{H} \alpha$ calibration relation (Kennicutt 1998) and assuming a case $\mathrm{B}$ recombination theory for the $\mathrm{Ly} \alpha / \mathrm{H} \alpha$ line ratio. Nevertheless, SFRs inferred from the UV continuum are found to be inconsistent with $\operatorname{SFR}(\operatorname{Ly} \alpha)$. It appears that $\operatorname{SFR}(\operatorname{Ly} \alpha)$ is typically lower than SFR(UV) by a factor of several (Ajiki et al. 2003; Taniguchi et al. 2005; Tapken et al. 2007; Gronwall et al. 2007). In principle, correction for internal reddening could reconcile these two indicators. Different extinctions experienced by the continuum and emission line radiation may, however, arise due to geometrical effects (Calzetti et al. 1994; Giavalisco et al. 1996); in addition, radiative transfer effects of the Ly $\alpha$ line imply that this issue is far from being resolved. In applying such a calibration, caution should therefore be taken. This need for caution is demonstrated further in cosmological studies where only a fraction of UV-selected galaxies show Ly $\alpha$ in emission (Shapley et al. 2003). Furthermore, some high- $z$ studies have unveiled very high rest frame Ly $\alpha$ equivalent widths ( $E W \mathrm{~s}$, Rhoads et al. 2003; Finkelstein et al. 2007) and it appears unlikely that such high $E W$ s can result from the ionizing output of a normal stellar population. In the case of an inhomogeneous ISM where dust is distributed in neutral gas clouds Neufeld (1991) and Hansen \& Peng Oh (2006) showed that Ly $\alpha$ photons could escape more easiy than continuum radiation. In this scenario, intrinsic Ly $\alpha E W$ s are expected to increase, which would explain the high values observed in those studies.

With respect to its importance for cosmology, many studies have attempted to understand the physical processes governing the fraction of escaping Ly $\alpha$ photons. Early observations at low redshift (Meier \& Terlevich 1981; Deharveng et al. 1985; Terlevich et al. 1993) indicated that Ly $\alpha$ was either far weaker than predicted or even absent from starburst galaxies. Initially, this weakening was attributed to dust attenuation, which was confirmed by a correlation observed between $E W_{\mathrm{Ly} \alpha}$ and metallicity (Charlot \& Fall 1993). These interpretations notwithstanding, Giavalisco et al. (1996) reached the opposite conclusion, finding no clear correlation between $E W_{\mathrm{Ly} \alpha}$ or $\mathrm{Ly} \alpha / \mathrm{H} \beta$ and $E(B-V)$. Furthermore, dust correction failed to reconcile the observed with the intrinsic $\mathrm{Ly} \alpha / \mathrm{H} \beta$ ratio predicted by case $\mathrm{B}$ recombination theory. Spectroscopic studies have outlined the Ly $\alpha$ observational puzzle. Kunth et al. (1994) and Thuan \& Izotov (1997), with the Goddard High Resolution Spectrograph (GHRS) and Space Telescope Imaging Spectrograph (STIS), have observed damped Ly $\alpha$ absorption in IZw 18 and SBS 0335052 , the most metal-deficient galaxies known at low- $z$. In the purely dust-regulated model, a prominent Ly $\alpha$ emission feature would be expected. On the other hand, Lequeux et al. (1995) detected strong Ly $\alpha$ emission in a far more metal- and dust-rich starburst Haro 2. Further studies have shed light on the mechanisms by which $\operatorname{Ly} \alpha$ photons may escape their host. Kunth et al. (1998) observed the Ly $\alpha$ morphology for 8 low- $z$ starbursts ranging from emission to absorption. They measured systematic blueshifts between the Ly $\alpha$ feature and Low ionization State (LIS) metal absorption features in the ISM when Ly $\alpha$ was observed in emission, indicative of an outflowing neutral medium. P Cygni profiles, with a redshifted emission peak with respect to the systemic velocity, were also found in these spectra. Furthermore, Mas-Hesse et al. (2003) applied hydrodynamic models (Tenorio-Tagle et al. 1999) in interpreting the different observed Ly $\alpha$ profiles as a function of starburst evolution and viewing geometry. They found that the Ly $\alpha$ emission visibility and shape were driven mostly by the kinematical configuration of the neutral gas. Over the past few years, theoretical studies and numerical simulations were developed for the same purpose. Ahn et al. (2003) and Verhamme et al. (2006) showed how the variety of Ly $\alpha$ profiles were created by the expansion of a superbubble of neutral gas and the properties of the ISM (HI column density and dust content). Hansen \& Peng Oh (2006) utilized the original idea of Neufeld (1991) to investigate the effects of a multi-phase ISM. More cosmological-orientated simulations (Tasitsiomi 2006) were completed, although the effects of dust remain to be treated. The simulations can now attempt to model arbitrary intrinsic emission characteristics, hydrogen density, velocity fields and dust distributions (Verhamme et al. 2006), and reproduce consistently the Ly $\alpha$ profiles observed in $z \sim 3$ LBGs (Verhamme et al. 2007; Schaerer \& Verhamme 2008).

The complex nature of the $\operatorname{Ly} \alpha$ escape probability revealed by low- $z$ spectroscopic studies rises further questions. The resonant scattering phenomenon of $\operatorname{Ly} \alpha$ line may cause $\operatorname{Ly} \alpha$ photons to travel and be emitted far from their production sites, and hence be spatially uncorrelated with non-resonant radiation $(\mathrm{H} \alpha$ or continuum photons). UV-targeted spectroscopic studies may therefore miss a significant fraction of the $\operatorname{Ly} \alpha$ emission if it is scattered away from the aperture. Ionized holes in the ISM may also allow the escape of Ly $\alpha$ photons in a spatially limited region and transmission may vary significantly on small scales across the starburst region. These considerations are the motivation for our Ly $\alpha$ imaging survey with the Advanced Camera for Survey (ACS) onboard the Hubble Space Telescope (HST). We observed a hand-picked sample of six nearby star forming galaxies to explore a large range of relevant parameters. Preliminary results were presented in Kunth et al. (2003); more detailed studies were published independently for ESO 338-04 (Hayes et al. 2005) and Haro 11 (Hayes et al. 2007). Emission and absorption were found on very small scales in central regions of the starburst, while absorption is observed in front of many of the brightest UV sources.

Besides the HST observations, we use ground-based observations from the ESO New Technology Telescope (NTT) and the Nordic Optical Telescope (NOT) to map $\mathrm{H} \alpha$ and $\mathrm{H} \beta$ emission and derive extinction from the Balmer decrement $(\mathrm{H} \alpha / \mathrm{H} \beta)$. Investigating correlations between $E(B-V)$ and Ly $\alpha$ emission enables us to disentangle the role of the dust from other parameters in the Ly $\alpha$ escape mechanism and investigate the implications of proper dust correction on high-redshift studies. The paper is structured as follows: in Sect. 2 we describe the observations and the data reduction, in Sect. 3 we present the results, Sect. 4 is dedicated to the discussion of these results, and finally in Sect. 5 we present our conclusions. We assume throughout this paper a cosmology of $H_{0}=72 \mathrm{~km} \mathrm{~s}^{-1} \mathrm{Mpc}^{-1}, \Omega_{\mathrm{M}}=0.3$ and $\Omega_{\Lambda}=0.7$.

\section{Observations and data reduction}

Our sample contains six local starburst galaxies that were handpicked to cover a range of relevant intrinsic parameters. Based 
Table 1. Targets general properties. $E(B-V)_{\mathrm{MW}}$ is the Galactic extinction given by Schlegel et al. (1998) and $M_{\mathrm{B}}$ magnitudes are in $\mathrm{AB}$ system. References: 1: Bergvall \& Östlin (2002), 2: Papaderos et al. (2006), 3: Gonzalez Delgado et al. (1998), 4: Izotov et al. (2001).

\begin{tabular}{lllllllll}
\hline \hline $\begin{array}{l}\text { Target } \\
\text { name }\end{array}$ & $\begin{array}{l}\text { Other } \\
\text { name }\end{array}$ & RA(2000) & Dec(2000) & $E(B-V)_{\text {MW }}$ & $z$ & $\begin{array}{l}12+ \\
\log (\mathrm{O} / \mathrm{H})\end{array}$ & $M_{\mathrm{B}}$ & Ref. \\
\hline Haro 11 & & & & & & & & \\
SBS 0335-052 & ESO 350-38 & $00: 36: 52.5$ & $-33: 33: 19$ & 0.049 & 0.020598 & 7.9 & -20 & 1 \\
IRAS 08339+6517 & PGS 0335-052E & $03: 37: 44.0$ & $-05: 02: 40$ & 0.047 & 0.013486 & 7.3 & -17 & 2 \\
Tololo 65 & ESO 380-27 & $08: 38: 23.2$ & $+65: 07: 15$ & 0.092 & 0.019113 & 8.7 & -21 & 3 \\
NGC 6090 & Mrk 496 & $12: 25: 46.9$ & $-36: 14: 01$ & 0.074 & 0.009 & 7.6 & -15 & 4 \\
ESO 338-04 & Tol 1924-416 & $19: 11: 40.7$ & $+52: 27: 24$ & 0.020 & 0.029304 & 8.8 & -21 & 3 \\
\hline
\end{tabular}

upon UV-properties, the selection covers a range of dust content, luminosity, and the variety of observed Ly $\alpha$ profiles: firstly, the sample consists of four Ly $\alpha$ emitters from Calzetti \& Kinney (1992), among which candidates representing a range in line profiles are selected (Kunth et al. 1998; Mas-Hesse et al. 2003). Two known Ly $\alpha$ absorbers are included from Thuan \& Izotov (1997). General information and properties of our targets are given in Table 1.

\subsection{HST observations}

Two general observer programmes, with the Hubble Space Telescope (HST), were devoted to observations of the six targets: GO9470 which uses the Solar Blind Channel (SBC) of the Advanced Camera for Surveys (ACS) to observe Ly $\alpha$ online (F122M) and continuum (F140LP), and GO10575 using the High Resolution Camera (HRC) and Wide Field Camera (WFC) to obtain $\mathrm{H} \alpha$, near-UV, and optical continuum broadband observations. Detailed description of these observations is presented in Östlin et al. (2008).

All images were drizzled using the MULTIDRIZZLE task in STSDAS package under NOAO/IRAF onto the same pixel scale $\left(0.025^{\prime \prime} \mathrm{pix}^{-1}\right)$ and the same orientation. Remaining shifts were corrected with GEOMAP and GEOTRAN, and cosmic rays removed using CREDIT task. The images for each target were then convolved to the same Point Spread Function (PSF) using DIGIPHOT/DAOPHOT, using the lowest quality PSF image as reference.

The production of continuum subtracted Ly $\alpha$ images from F122M (online) and F140LP (offline) is untrivial and requires sophisticated techniques for many reasons. The effective wavelength of the continuum filter is rather far from the online filter $(\Delta \lambda / \lambda \simeq 0.22)$ and the UV continuum between the two filters deviates significantly from a power law $\left(f_{\lambda} \propto \lambda^{\beta}\right)$ and is sensitive to age and $E(B-V)$. By neglecting these considerations and describing the behavior of the UV continuum near Ly $\alpha$ with a power law, we would obtain results ranging from absorption to strong emission according to the values set to $\beta$. Preliminary results, by Kunth et al. (2003), pointed out these limitations and the need for an elaborated subtraction method. Subsequently, Hayes et al. (2005) and, in more detail, Hayes et al. (2008) presented a reliable extrapolation from F140LP to F120M described by the Continuum Throughput Normalization factor (CTN). To estimate the CTN in each pixel, all images from F122M to F814W are used to fit the Starburst99 spectral evolutionary models (Leitherer et al. 1999; Vázquez \& Leitherer 2005); the method uses filters that sample the UV continuum slope and the $4000 \AA$ break to fit burst age and stellar $E(B-V)$ using $\chi^{2}$ minimisation. The nebular contribution and the stellar components are then treated independently. The $\mathrm{H} \alpha$ image is used to constrain the nebular gas contribution to the total SED, while $V$ and $I$ images allow us to estimate the stellar part. The gas spectrum is subtracted and age and mass are fitted in two stellar components.

To generate Starburst 99 models, we set the metallicity as derived from observations of each object: for IRAS $08339+6517$ and NGC $6090 Z=0.02$, for Haro 11 and ESO 338-04 $Z=0.004$, and for SBS 335-052 and Tololo $65 Z=0.001$. Although Hayes et al. (2008) show that an error below 50\% on the metallicity does not affect significantly the continuum subtraction, the metallicity estimate remains the factor driving the accuracy of the subtraction method.

A standard Salpeter (1955) IMF is used ( $\alpha=2.35, \mathrm{~d} N=$ $\left.M^{-\alpha} \mathrm{d} M\right)$ in the range $0.1 M_{\odot}$ to $120 M_{\odot}$. Multi-stellar component fitting, used for continuum subtraction, is almost completely insensitive to the IMF slope and mass range. Finally, an instantaneous burst scenario is adopted because it is more appropriate for individual pixels but the choice of a constant star formation scenario would not affect global photometry of the galaxies or the contribution of the underlying stellar population.

\subsection{Ground-based observations}

The southern targets of our sample (cf. Table 2) were observed using the New Technology Telescope (NTT) at La Silla (ESO) during the nights of 18, 19, and 20 September 2004 (apart from Tololo 65, which was observed in service mode on 28 January 2003). Narrow-band imaging was performed for all targets in $\mathrm{H} \alpha, \mathrm{H} \beta$, and [OIII] $\lambda 5007 \AA$, and the nearby continuum of each line. The first night of the run, 18th Sept, the seeing was consistently below $1.2^{\prime \prime}$, yet the presence of thin cirrus prevents a direct calibration using standard stars. Observational conditions on the night of 19 Sept. were excellent: photometric sky and sub-arcsec seeing. On the final night, the seeing was poorer, exceeding $2^{\prime \prime}$, although the photometric quality was still good. Spectrophotometric standard stars Feige110, LDS749B, GD50, and GD108, selected from the Oke (1990) catalog, were observed at regular intervals during each night and in each filter. Both the ESO Multi-Mode Instrument (EMMI) (Dekker et al. 1986) and Super Seeing Imager 2 (SuSI2) (D'Odorico et al. 1998) were used interchangeably. A binning of $2 \times 2$ pixels was used, providing plate-scales of $0.332^{\prime \prime} \mathrm{pix}^{-1}$ and $0.161^{\prime \prime} \mathrm{pix}^{-1}$, and fields-of-view of $9.1 \times 9.9^{\prime}$ and $5.5 \times 5.5^{\prime}$ for EMMI-R and SuSI 2 respectively. NTT observations are summarized in Table 2 with ESO filter codes. The good seeing observations of the night Sept. 18, 2004 are calibrated using secondary standard stars in the field from the photometric night 19 and/or Sept. 20, 2004.

The remaining northern targets (IRAS 08339+6517 and NGC 6090) were observed with the Nordic Optical Telescope (NOT) at La Palma during the nights of 14, 15, and 16 February 
Table 2. Ground-based observations of our six targets. Northern targets have been observed with the Nordic Optical Telescope and southern ones with ESO New Technology Telescope. $\mathrm{H} \alpha, \mathrm{H} \beta$ online and respective continuum observations are listed with the instrument and filter name and the exposure time (in seconds) in each band.

\begin{tabular}{|c|c|c|c|c|c|c|c|c|}
\hline \multirow{2}{*}{$\begin{array}{l}\text { Target } \\
\text { ESO/NTT observations }\end{array}$} & \multicolumn{2}{|c|}{$\mathrm{H} \alpha$} & \multicolumn{2}{|c|}{$\mathrm{H} \alpha$ continuum } & \multicolumn{2}{|c|}{$\mathrm{H} \beta$} & \multicolumn{2}{|c|}{$\mathrm{H} \beta$ continuum } \\
\hline & Camera/Filter & Exp. & Camera/Filter & Exp. & Camera/Filter & Exp. & Camera/Filter & Exp. \\
\hline Haro 11 & EMMI-R/598 & 900 & EMMI-R/597 & 1200 & SuSI2/549 & 2866 & EMMI-R/770 & 1800 \\
\hline SBS 0335-052 & EMMI-R/598 & 1800 & EMMI-R/596 & 4800 & SuSI2/548 & 900 & EMMI-R/771 & 4500 \\
\hline ESO 338-04 & EMMI-R/597 & 1800 & SuSI2/778 & 3600 & SuSI2/719 & 2400 & EMMI-R/771 & 4500 \\
\hline Tololo 65 & SuSI2/709 & 1200 & SuSI2/778 & 1200 & SuSI2/719 & 3600 & SuSI2/717 & 1800 \\
\hline NOT observations & & & & & & & & \\
\hline IRAS 08339+6517 & ALFOSC/70 & 2400 & ALFOSC/78 & 3000 & ALFOSC/113 & 5000 & ALFOSC/17 & 3300 \\
\hline NGC 6090 & ALFOSC/53 & 4200 & ALFOSC/78 & 2400 & ALFOSC/40 & 3600 & ALFOSC/17 & 2100 \\
\hline
\end{tabular}

2004, using the Andalucia Faint Object Spectrograph and Camera (ALFOSC). Unfortunately, the observational conditions were not photometric during the observing run. For NGC 6090, we used observations taken on March 28th 2006 under photometric conditions. The calibration was performed by adopting BD33 as the standard star. Our science images were then calibrated using field stars from the 2006 observations in each filter. For IRAS 08339+6517, we made use of HST images for the same purpose. Firstly, calibrated $\mathrm{H} \alpha$ images were rescaled to the ALFOSC pixel-plate $\left(0.19^{\prime \prime}\right.$ pix $\left.^{-1}\right)$. Aperture photometry was then obtained and used for the calibration of the groundbased $\mathrm{H} \alpha$ observations. The $\mathrm{H} \beta$ continuum images were calibrated using rescaled $\mathrm{F} 435 \mathrm{~W}$ images and $\mathrm{H} \beta$ online images were then calibrated with respect to the continuum images using photometry of several stars in the field of view. Global estimates for IRAS $08339+6517$ are the least accurate of the galaxy sample due to the less-than-ideal calibration of $\mathrm{H} \beta$. However, pixel-topixel measurements suffer from similar uncertainties, therefore pixel-to-pixel trends are as reliable as they are in any other target, irrespective of whether the value of $E(B-V)$ is perfect.

Data were first reduced using standard NOAO/IRAF procedures. Images were bias-subtracted, and flat-field corrected using well exposed sky and dome flats. Images were registered using field stars and the GEOMAP and GEOTRAN tasks, and images from the same bands were coadded with inverse variance weighting. Finally, reduced frames for each target were smoothed to the PSF of the lowest quality seeing image using GAUSS.

$\mathrm{H} \alpha$ images were corrected for [NII] contamination and both $\mathrm{H} \alpha$ and $\mathrm{H} \beta$ images corrected for underlying stellar absorption. [NII] contamination was estimated from NII $\lambda 6583 \AA / \mathrm{H} \alpha$ found in literature (see Table 3 ) and the ratio NII $\lambda 6584 \AA / N I I \quad \lambda 6548 \AA \simeq 3$ (Osterbrock 1989).

Stellar absorption was estimated using the best-fitting Starburst 99 spectrum given by the procedure described in Sect. 2.1. The most updated available stellar atmosphere models were used with Starburst99 stellar libraries (Martins et al. 2005), including full line-blanketing, to obtain the most accurate estimate of equivalent width for the $\mathrm{H} \alpha$ and $\mathrm{H} \beta$ stellar absorption features.

Finally, using the Balmer decrement $\mathrm{H} \alpha / \mathrm{H} \beta$ and assuming a case B intrinsic line ratio of 2.86 (Osterbrock 1989) at a temperature of $10000 \mathrm{~K}$, we derived the $E(B-V)$ in the gas phase using the parameterization of Cardelli et al. (1989) and the equation:

$E(B-V)_{\mathrm{H} \alpha / \mathrm{H} \beta}=\frac{2.5 \times \log \left(2.86 / R_{\mathrm{obs}}\right)}{k\left(\lambda_{\alpha}\right)-k\left(\lambda_{\beta}\right)}$
Table 3. $\mathrm{H} \alpha$ correction for NII contamination with references where the ratio has been taken from.

\begin{tabular}{|c|c|c|}
\hline Target & $\frac{[\mathrm{NIII]} \lambda 6583 \AA}{\mathrm{H} \alpha}$ & Reference \\
\hline Haro 11 & 0.189 & Bergvall \& Östlin (2002) \\
\hline ESO 338-04 & 0.031 & Bergvall \& Östlin (2002) \\
\hline NGC 6090 & 0.411 & Moustakas \& Kennicutt (2006) \\
\hline IRAS $08339+6517$ & 0.25 & Margon et al. (1988) \\
\hline SBS 0335-052 & 0.003 & Izotov et al. (1997) \\
\hline Tololo 65 & 0.005 & Izotov et al. (2004) \\
\hline
\end{tabular}

where $R_{\mathrm{obs}}=f_{\mathrm{H} \alpha} / f_{\mathrm{H} \beta}$ is the absolute observed flux ratio, and $k\left(\lambda_{\alpha}\right), k\left(\lambda_{\beta}\right)$ are the extinction curves at $\mathrm{H} \alpha$ and $\mathrm{H} \beta$ wavelengths respectively. According to Cardelli et al. (1989), $k\left(\lambda_{\alpha}\right) \sim 2.63$ and $k\left(\lambda_{\beta}\right) \sim 3.71$.

\subsection{Uncertainties}

The calibration of individual images in $\mathrm{H} \alpha$ and $\mathrm{H} \beta$ lines are affected by the typical sources of noise and uncertainty during the data reduction procedure. The principal source of uncertainty originates in the flux measurement of the calibration standard stars. This accounts for about 5\% in our observations. Errors of approximatively few percent may be produced by other effects, such as residuals in the flat-field corrections, residuals in the sky background, and continuum subtraction. We assume that all of these effects produce an error in the flux measurement of approximatively $10 \%$. We produce extinction maps by dividing $\mathrm{H} \alpha$ images into $\mathrm{H} \beta$ images, and, for some galaxies, these images were obtained with different instruments (i.e. different plate-scales and orientations); the errors in image alignment and registration may lead to quite significant uncertainties, since we aim, in our science analysis, to investigate the Ly $\alpha$ and dust amount variations on a pixel scale. The procedure to estimate the impact of such misalignments was the following.

The rms of the alignment fit provided by GEOMAP is about 0.2 pixel. Consequently, to estimate the misalignment (and only to that purpose), we rebinned $\mathrm{H} \alpha$ and $\mathrm{H} \beta$ images to a new pixel size, $1 / 5$ of the original pixel size. We then created new images with artificial shifts of $+1,0$ or -1 pixel in $x$ and $y$ directions. This provided a 9-image data cube for both $\mathrm{H} \alpha$ and $\mathrm{H} \beta$. Using all combinations of $\mathrm{H} \alpha$ and $\mathrm{H} \beta$ shifted images, we derived the ratio $\mathrm{H} \alpha / \mathrm{H} \beta$ and constructed an $E(B-V)$ data cube. We eventually computed the standard deviation of the extinction, $\sigma_{E(B-V)}$, at each pixel $[x, y]$. 
Misalignment errors were also a concern for the Ly $\alpha$ image with respect to $\mathrm{H} \alpha$ and online images with respect to continuum ones. These could produce errors in the $\mathrm{Ly} \alpha / \mathrm{H} \alpha$ ratio and the equivalent widths. The estimation of these quantities takes account of this uncertainty. We accounted for uncertainties in the Ly $\alpha$ continuum subtraction procedure by assuming a statistical error of $10 \%$ of the Ly $\alpha$ flux. This uncertainty depends, in reality, on the signal-to-noise in each resolution element where the continuum is fitted, and the value adopted here corresponds to $S / N=5 \%$. The final error bars are shown in the scatter plots in Sect. 3. For the sake of readability, we plot the mean errors instead of the individual errors of each point. When the emission is dominated by the conglomerate points of the diffuse halo (which is the case for most of the plots), we show the mean computed between only the maximum and minimum values, to cover the full range of error variations.

\section{Analysis}

\subsection{Individual galaxies}

We describe in this section our imaging results and perform a detailed analysis for each individual object of our sample. We examine the potential correlations between Ly $\alpha$ and the different physical parameters on a pixel scale. This allow us to tackle the complex physics of $\operatorname{Ly} \alpha$ radiation.

\subsubsection{HARO 11}

The $\mathrm{H} \alpha$ image in Fig. 1 in the first column shows a complex morphology with three main star-forming condensations (Kunth et al. 2003). The continuum subtracted $\operatorname{Ly} \alpha$ image does not delineate this morphology, showing $\operatorname{Ly} \alpha$ in emission in only knot $\mathrm{C}$, whereas it is seen in absorption in knot $\mathrm{A}$ and $\mathrm{B}$. The decoupling of $\operatorname{Ly} \alpha$ from the continuum is clearly observable in the bottom frame, which represents a Ly $\alpha$ map at HST resolution overlayed by FUV (1500 ̊) contours. The emission exhibits two different components, consisting of a central bright knot and a low surface brightness diffuse emission. By examining the extinction map, it appears that the diffuse component is not regulated by the amount of dust. Moreover, the bright Ly $\alpha$ emission in knot $\mathrm{C}$, corresponds to a high extinction region.

This galaxy is a well known Ly $\alpha$ emitter (Kunth et al. 1998), while the detection of Lyman continuum leakage by Bergvall et al. (2006) is still controversial (Grimes et al. 2007). It was studied in more detail by Hayes et al. (2007).

\subsubsection{ESO 338-04}

In the second column of Fig. 1, the Ly $\alpha$ image shows three main absorption regions and a surrounding bright emission. The absorption sites correspond to relatively dusty regions of the galaxy seen in the extinction map, which traps Ly $\alpha$ photons, while the emission is not correlated with the dust content. The last component is, again, the diffuse emission around and overall the galaxy, with a low surface brightness, which corresponds to the resonant decoupling of Ly $\alpha$ photons. Many dust features are clearly visible in the $E(B-V)$ map with a clumpy-like structure, which follows roughly the $\mathrm{H} \alpha$ structure.

The Ly $\alpha$ image is produced by matching the HST/ACS image to the NTT resolution. This process disperses the light from the central absorption region to more extended regions, reveals small absorption features, and dims the surface brightness of the emission component. The bottom frame shows again how Ly $\alpha$ is uncorrelated with the FUV continuum, which traces unobscured star formation sites.

\subsubsection{NGC 6090}

The third column in Fig. 1 indicates that the interacting system NGC 6090 exhibits Ly $\alpha$ emission from each component, at a distance of about $6^{\prime \prime}$ from each other. The emission peaks around low extinction regions and the overlapping region between the two components appears very dusty. The extinction map of NGC 6090 illustrates dust pattern similar to that of a spiral structure that has no evident correlation with the ionized gas traced by $\mathrm{H} \alpha$ emission.

The main $\mathrm{H} \alpha$ structures correspond to the Ly $\alpha$ emission components, although the largest Ly $\alpha$ emission represents a small region in $\mathrm{H} \alpha$, and vice-versa. This discrepancy may be due to the large amount of dust in the upper left component that could destroy a significant fraction of Ly $\alpha$ photons. Knot A appears also dustier $\left(E_{B-V, \text { gas }} \sim 0.75\right)$ than knot B $\left(E_{B-V, \text { gas }} \sim 0.55\right)$.

\subsubsection{IRAS $08339+6517$}

This nuclear starburst shows a spiral structure that is conspicuous in the $\mathrm{H} \alpha$ (Fig. 2) and FUV continuum images, the latter of which is not shown here). However the Ly $\alpha$ image does not resemble any other image, showing central bright emission and an ubiquitous halo component. The FUV contours exhibit far more detail in the arms of the galaxy, which consist of many star clusters for which $\operatorname{Ly} \alpha$ is absent.

In the extinction map, a dust-free central spot is clearly evident. The dust distribution has no clear relationship with the emission maps.

\subsubsection{SBS $335-052$}

The dust distribution appears to be correlated with the $\mathrm{H} \alpha$ emission of this galaxy. In Fig. 2, the brightest region in $\mathrm{H} \alpha$ corresponds to the most significant concentration of dust. A shell structure and dust-free region toward the S-E of the bright region are also visible in both images. The bright dusty spot corresponds to a relatively high Ly $\alpha$ absorption region. The galaxy shows Ly $\alpha$ only in absorption surrounded by faint diffuse emission. Despite the significant $\mathrm{H} \alpha$ or FUV continuum emission, it appears that no Ly $\alpha$ photons escape directly without scattering on neutral hydrogen.

\subsubsection{Tololo 65}

In the rightmost column of Fig. 2, no Ly $\alpha$ structure is observed for this galaxy. Athough the HST image indicates some emission features (bottom frame), they have been smoothed to the NTT resolution. Degrading the resolution of an HST image can produce ghost features since the emission becomes as weak as and can no longer be distinguished from the background level. These artefacts in the background, although less dramatic, are also present in other galaxies. The $\mathrm{H} \alpha$ map presents two components emission that resembles the structure of the dust content in the $E(B-V)$ map.

To investigate Ly $\alpha$ emission variations and possible correlations with dust or other parameters on the smallest possible scale, we produced scatter plots for the images. In the extinction maps presented in Fig. 1, each point represents one pixel in the galaxy region that has been isolated by masking the background 

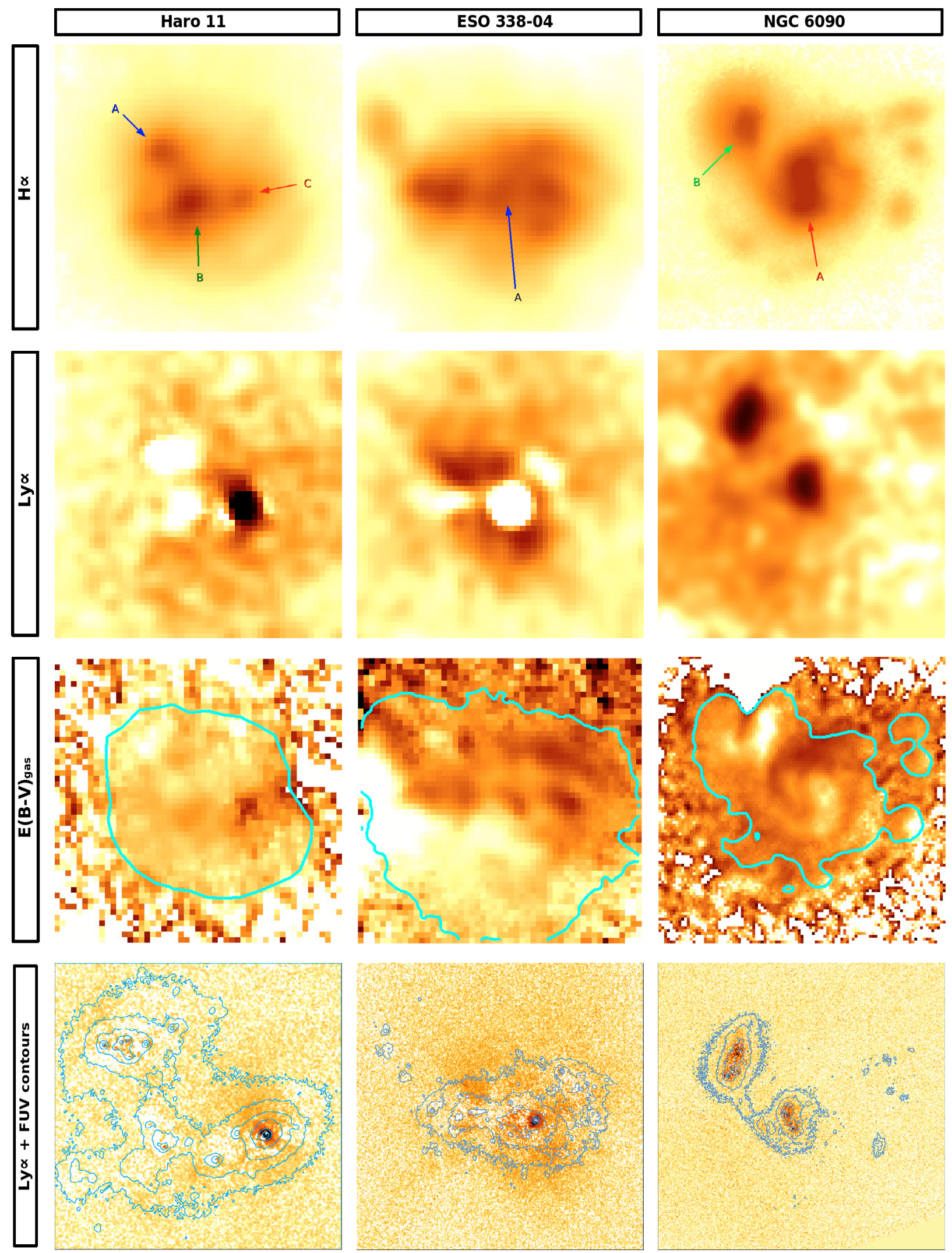

Fig. 1. Galaxy imaging (Part 1): from top to bottom: $\mathrm{H} \alpha \mathrm{Ly} \alpha, E(B-V)_{\text {gas }}$ map, and Ly $\alpha$ as seen by HST overlayed with FUV contours. Inverted logarithmic scale is used, showing emission in black and absorption in white. The extinction map is overlayed with the mask generated following the description in Sect. 4. Regions out of the contour are excluded from our study. The dustiest regions are in black. From left to right with the FoV and spatial scale in parentheses: ground-based images (top three rows): Haro 11 (17", $\left.0.4 \mathrm{kpc} /{ }^{\prime \prime}\right)$, ESO 338-04 (17", $\left.0.2 \mathrm{kpc} /{ }^{\prime \prime}\right)$, and NGC 6090 $\left(19^{\prime \prime}, 0.57 \mathrm{kpc} /{ }^{\prime \prime}\right)$; HST images (last row): Haro 11 (13"), ESO 338-04 (15"), and NGC $6090\left(21^{\prime \prime}\right)$. North is down and East to the right. 

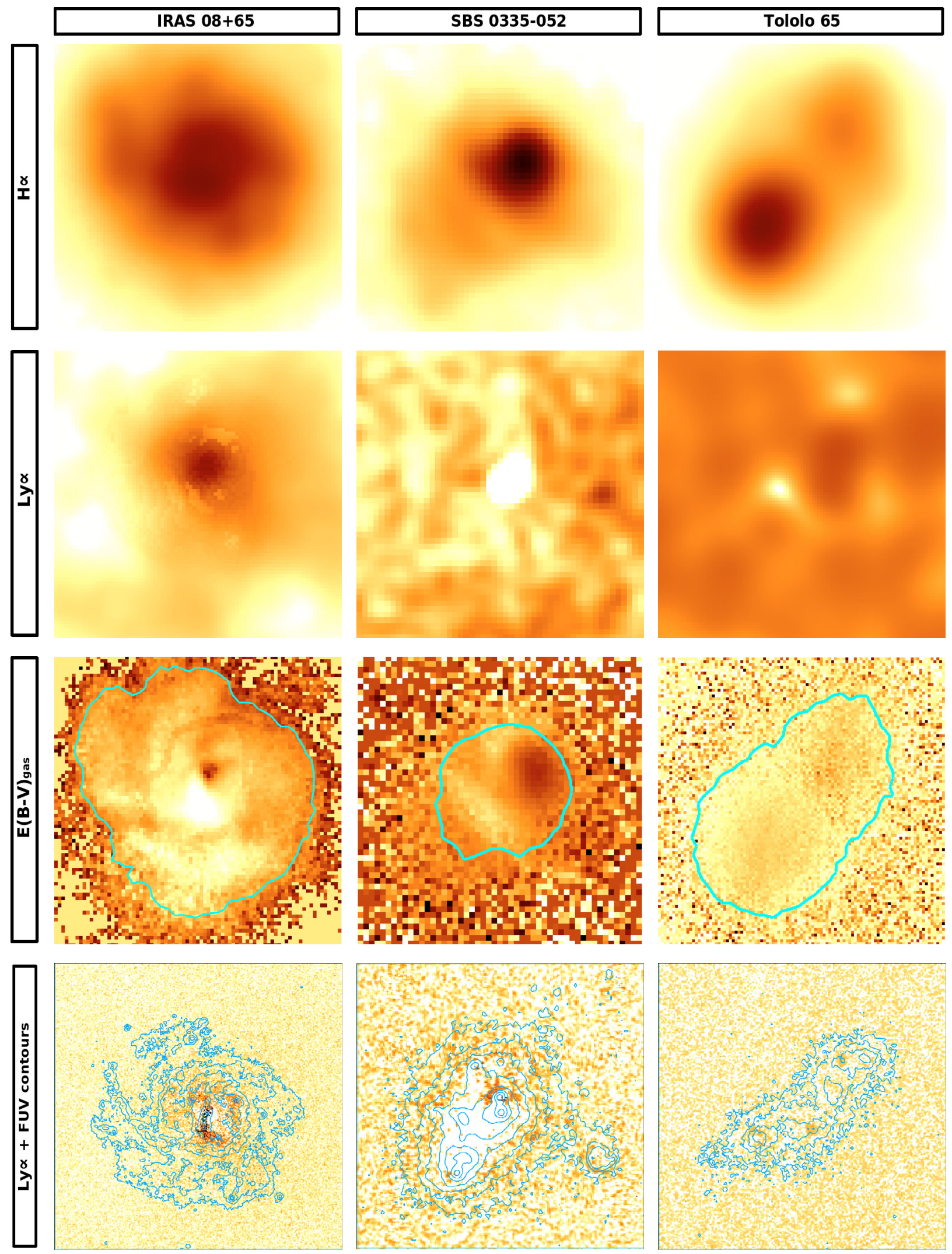

Fig. 2. Galaxy imaging (Part 2): from top to bottom: $\mathrm{H} \alpha$, $\mathrm{Ly} \alpha, E(B-V)_{\text {gas }}$ map, and Ly $\alpha$ as seen by HST overlayed with FUV contours. Inverted logarithmic scale is used, showing emission in black and absorption in white. The extinction map is overlayed with the mask generated following the description in Sect. 4. Regions out of the contour are excluded from our study. The dustiest regions are in black. From left to right with the FoV in parentheses: ground-based images (top three rows): IRAS 08339+6517 (15", $\left.0.38 \mathrm{kpc} /{ }^{\prime \prime}\right)$, SBS 335-052 (4", $\left.0.27 \mathrm{kpc} /{ }^{\prime \prime}\right)$, Tololo 65 ( $8^{\prime \prime}$, $\left.0.18 \mathrm{kpc} /{ }^{\prime \prime}\right)$; HST images (last row): IRAS 08339+6517 (14"), SBS 335-052 (5"), Tololo $65\left(8^{\prime \prime}\right)$. North is up and East to the left. 

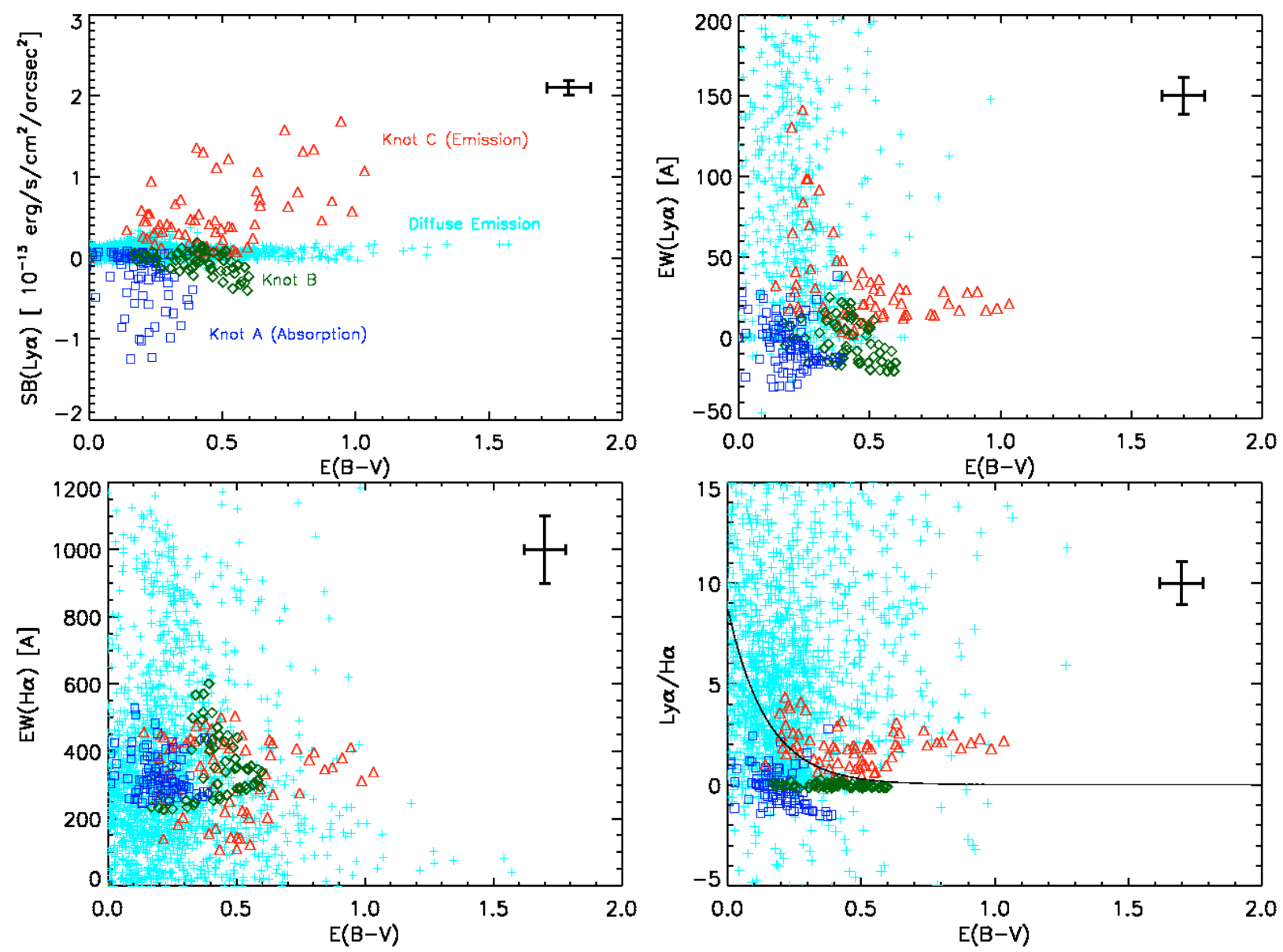

Fig. 3. Haro 11 scatter plots. Top-left: Ly $\alpha$ surface brightness as a function of the extinction determined from the Balmer decrement. Regions of knots A, B, C and the diffuse emission are marked in the figure and represented with different colors and different symbols: emission is in red, absorption is in blue (knot A) and green ( knot B), and the diffuse emission component is in cyan. For the remaining plots, the same color code is used for the respective regions. Top-right: Ly $\alpha$ equivalent width vs. $E(B-V)$. Bottom-left: H $\alpha$ equivalent width vs. E(B - V). Bottom-right: $\mathrm{Ly} \alpha / \mathrm{H} \alpha$ ratio vs. $E(B-V)$. Overplotted on all figures the error bars corresponding to the uncertainties estimated in Sect. 2.3 .

at the $5 \sigma$ level. Regions of interest, such as star-forming knots and emission or absorption features, are highlighted using circular apertures and represented with different colors and symbols on the figures.

\subsection{Blended emission and absorption systems}

Haro 11 - The first plot in Fig. 3 presents the correlation between the Ly $\alpha$ emission and the extinction determined from the Balmer decrement tracing the dust in the gas phase $\left(E_{B-V, \mathrm{gas}}\right)$. The color-code represents different regions of interest consisting of circular apertures centered on the three main knots of the galaxy, which are marked on the Ly $\alpha$ image. We can see a diffuse emission component extending up to $E_{B-V} \sim 1.5$. Knot $\mathrm{C}$ shows a bright, dispersed emission with a mean extinction of 0.48 , while the absorption is localized essentially around knots A and B. The mean extinctions in each knot are derived using the ratio of integrated $\mathrm{H} \alpha$ and $\mathrm{H} \beta$ fluxes within the corresponding apertures.

The presence of two distinct emission components indicates that two different physical processes control the escape of Ly $\alpha$ photons. Firstly, there is the diffuse component that shows the resonant decoupling of $\operatorname{Ly} \alpha$ photons scattered resonantly by hydrogen atoms until they escape far away from their production sites (and therefore, experiencing a significant range of

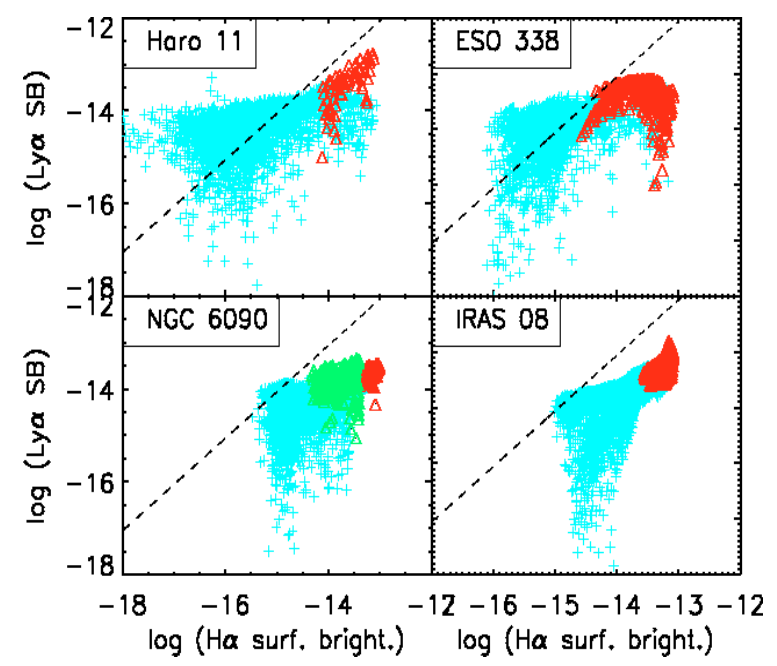

Fig. 4. Pixel distribution of the $\mathrm{Ly} \alpha$ against $\mathrm{H} \alpha$ surface brightness. A logarithmic scale is used and hence shows only positive contribution (emission). The dashed line represents the case B recombination ratio. The galaxy name is given in each plot.

extinction). On the other hand, the emission from knot $\mathrm{C}$ is more concentrated and represents photons escaping directly from this small region with a mean extinction $E_{B-V} \sim 0.48$. The Ly $\alpha$ resonant decoupling is also visible in Fig. 4, which illustrates 

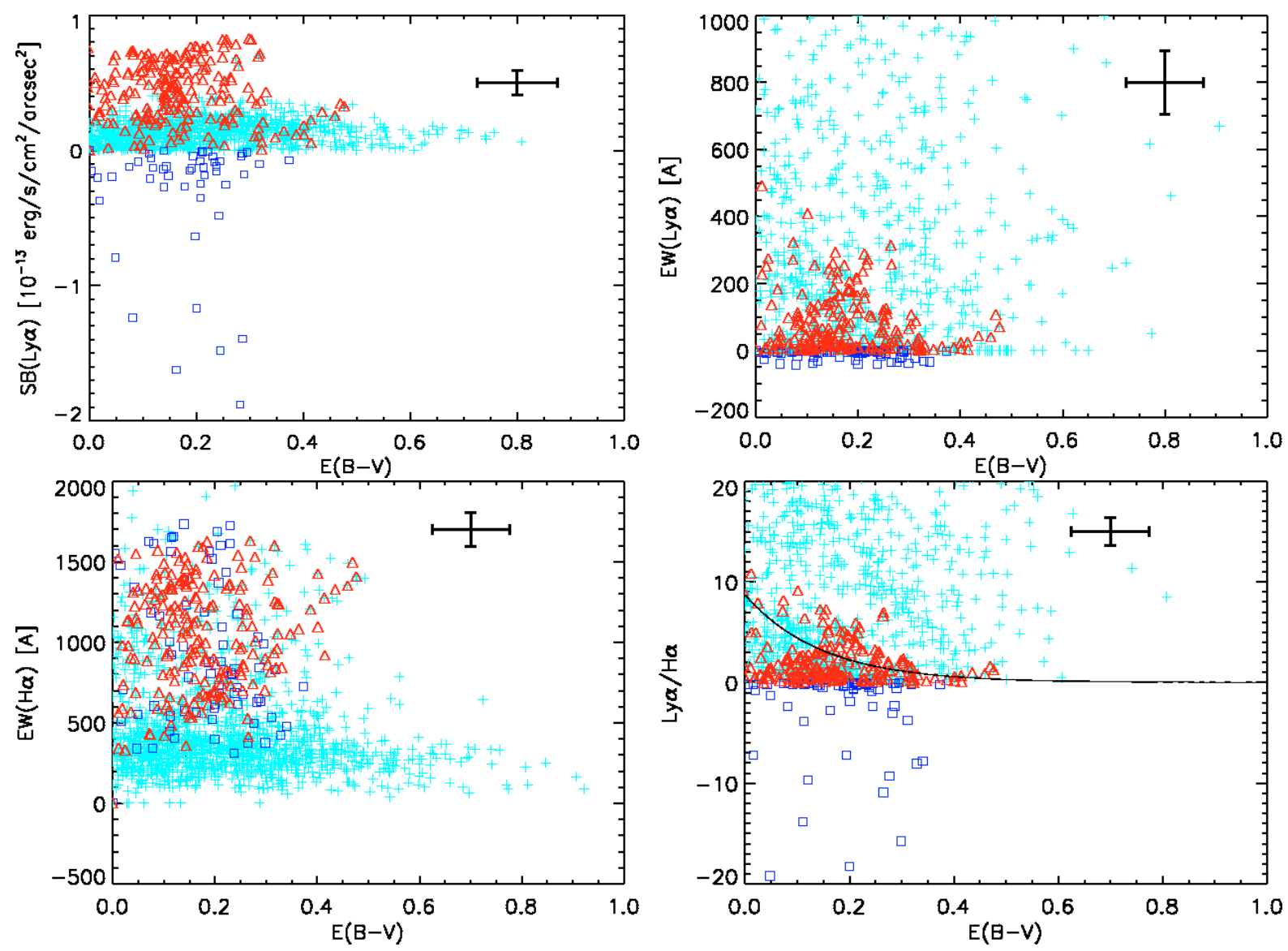

Fig. 5. ESO 338-04 scatter plots. Top-left: Ly $\alpha$ surface brightness vs. $E(B-V)$. Emission from the central region is in red, Absorption in the central region (knot A) is represented in blue (knot A), the surrounding emission is in red, and the diffuse emission component is in cyan. Same color code applied in all the plots. Top-right: $\mathrm{Ly} \alpha$ equivalent width vs. $E(B-V)$. Bottom-left: $\mathrm{H} \alpha$ equivalent width vs. $E(B-V)$. Bottom-right: $\mathrm{Ly} \alpha / \mathrm{H} \alpha$ ratio vs. $E(B-V)$. Error bars correspond to the uncertainties estimated in Sect. 2.3.

the correlation between the $\mathrm{Ly} \alpha$ and the $\mathrm{H} \alpha$ fluxes in $\log$-scale and therefore showing only positive pixels (in emission). We observe a first component at a low and almost constant Ly $\alpha$ surface brightness (around 10-14 $\mathrm{erg} \mathrm{s}^{-1} \mathrm{~cm}^{-2} \operatorname{arcsec}^{-2}$ ), independent of $\mathrm{H} \alpha$ emission. Due to their resonant scattering on HI atoms, Ly $\alpha$ photons reach regions where non-resonant photons, such as $\mathrm{H} \alpha$, are absent, which increase the $\mathrm{Ly} \alpha / \mathrm{H} \alpha$ ratio to higher than the Case B level (represented by a dashed line in the figure). The second component at higher $\operatorname{Ly} \alpha$ and $\mathrm{H} \alpha$ fluxes $\left(f_{\mathrm{Ly} \alpha} \geq 10^{-14} \mathrm{erg} \mathrm{s}^{-1} \mathrm{~cm}^{-2} \operatorname{arcsec}^{-2}\right)$ is always below the predicted recombination value. These pixels represent regions where $\mathrm{Ly} \alpha$ photons escape directly from their production site where $\mathrm{H} \alpha$ is also produced.

It is interesting that we observe Ly $\alpha$ in emission from knot C with $E_{B-V} \sim 0.48$, while only absorption is detected in knots A and $\mathrm{B}$, which have $E_{B-V} \sim 0.2$ and 0.41 , respectively. Indeed Ly $\alpha$ photons escape regions at higher extinction than those corresponding to pure absorption. The dust content is clearly not the main driver in the escape process of Ly $\alpha$ photons. A significant cover of static HI column density in knot A and an expanding neutral ISM and/or ionized HII holes in knot $\mathrm{C}$ may produce this observation.

By studying the Ly $\alpha$ equivalent width, we observe that the diffuse emission shows relatively high $E W_{\mathrm{Ly} \alpha}$, whereas the pure emission equivalent width in knot $\mathrm{C}$ (in red) is far weaker. This suggests that hard FUV radiation could create ionized holes through which $\operatorname{Ly} \alpha$ photons may escape, in a inhomogeneous distribution of HI and dust. In this case of multi-phase ISM, it has been shown (Neufeld 1991; Hansen \& Peng Oh 2006) that, due to their scattering off the dusty HI clumps, Ly $\alpha$ escapes in an easier way than non-resonant photons. We also observe that the diffuse emission (in cyan) corresponds to the highest equivalent width observed ( $E W_{\mathrm{Ly} \alpha}$ higher than $200 \AA$ ), since it represents photons that have been scattered far away from their production sites and escaped regions where the Ly $\alpha$ continuum is lower. This decline in emission is, again, symptomatic of the resonant nature of Ly $\alpha$ photons. Indeed, when we plot the equivalent width of $\mathrm{H} \alpha$ against extinction, we do not observe a correlation, which we would expect for non-resonant lines, since the online and continuum photons are regulated by the same physical processes, which are controlled by the dust content. The last figure shows how the $\operatorname{Ly} \alpha / \mathrm{H} \alpha$ ratio evolves according to the amount of dust. With a classical perspective, we expect to have an exponential decline represented by the dark curve when considering only selective extinction at two wavelengths and a case B intrinsic ratio of 8.7 (Brocklehurst 1971). The resonant nature of the $\operatorname{Ly} \alpha$ photons produces a different result. We observe a high dispersion for the halo component and an emission from knot $\mathrm{C}$ above the predicted level at higher extinction, which might support the view of scattering in an inhomogeneous ionized ISM that favors preferentially the escape of Ly $\alpha$ photons.

ESO 338-04 - This galaxy shows a similar pixel distribution in Ly $\alpha-E_{B-V}$ space to that of Haro 11 (Fig. 5). We see both emission and pure absorption features at, approximately, the same 
extinction (mean $E_{B-V} \sim 0.22$ and 0.23 for absorption and global emission respectively). The different regions are marked in the scatter plots with different colors consisting of a central absorption (in blue) surrounded by emission features (red). We also observe a halo of diffuse emission surrounding the starburst regions and independent of the extinction. However, the diffuse emission does not exceed $5 \times 10^{-14} \mathrm{erg} \mathrm{s}^{-1} \mathrm{~cm}^{-2} \operatorname{arcsec}^{-2}$ and accounts for about $70 \%$ of the total $\operatorname{Ly} \alpha$ emission from the galaxy. The same value was found by Hayes et al. (2005), although a different masking was used.

The absorption is seen only in the knot A (following the nomenclature of Hayes et al. 2005), superimposed on a region with an average extinction of $E_{B-V} \sim 0.2$ (mean extinction calculated, as for Haro 11, using integrated $\mathrm{H} \alpha$ and $\mathrm{H} \beta$ fluxes over this knot). Using longslit spectroscopy measurement of $\mathrm{H} \alpha / \mathrm{H} \beta$ in which the slit was positioned along the East-West direction), Östlin et al. (2003) found $E(B-V)$ values within a range comparable to our result $\left(E_{B-V \text {,gas }} \sim 0-0.25\right)$, apart from toward the east edge of the galaxy where a dustier region is seen in our extinction map $\left(E_{B-V \text {,gas }} \sim 0.4\right)$. We measure the same values of extinction in the surrounding regions as for knot $\mathrm{A}$, where $\operatorname{Ly} \alpha$ is found in emission. There is a trend of decline in the emission (though, with high dispersion) (until $E_{B-V} \sim 0.6$ ). The radiative transfer of $\operatorname{Ly} \alpha$ photons in a static (or almost static) ISM may produce the present situation: in the central region (knot A), Ly $\alpha$ photons are produced and immediately absorbed by neutral hydrogen. They are re-emitted according to space and frequency redistribution probability. Eventually, Ly $\alpha$ photons scatter resonantly in the wings until they reach a frequency that is sufficiently far away from the line center (where the absorption probability is close to unity) to be able to escape from the neutral medium. This also leads to a diffusion in space, which explains the emission seen around the central absorption at the same extinction. This effect is also seen in Haro 11 at HST resolution (Fig. 1), but does not appear at the NTT resolution due to smoothing effects. As shown by Ly $\alpha$ radiative transfer models (e.g. Verhamme et al. 2006), it seems that Ly $\alpha$ photons do not easily escape from their site of origin but scatter in space and frequency avoiding absorption by $\mathrm{HI}$ atoms at line center $\left(v_{0}=2.46 \times 10^{15} \mathrm{~Hz}\right)$.

In Fig. 5 we note a high $\operatorname{Ly} \alpha$ equivalent width, ranging from $-10 \AA$ for absorption (blue measurement) to $\sim 250 \AA$ in the surrounding emission (red measurement). Such high measurement of $E W$ for emission may be due to either the resonant scattering mechanism, which enables Ly photons to travel when continuum photons cannot, or a multi-phase ISM configuration, for which Hansen \& Peng Oh (2006) noted that, for a reasonable HI column density and amount of dust, the continuum is preferentially extinguished, boosting the initial $E W$ measurement easily by a factor of 2-5. This configuration may also explain the high $E W_{\mathrm{Ly} \alpha}$ observed in some high-redshift galaxies, extending up to $150 \AA$ in LALA $z \sim 5.7$ sources (Rhoads et al. 2003) in the case of spectroscopically confirmed candidates (higher possible equivalent widths were found in the imaging survey). On the other hand, $\mathrm{H} \alpha$ equivalent width map shows similar distribution to that of Haro 11 without any correlation.

The last frame shows the evolution of the $\mathrm{Ly} \alpha / \mathrm{H} \alpha$ ratio with extinction. In the central region around knot $\mathrm{A}, \mathrm{Ly} \alpha / \mathrm{H} \alpha$ follows loosely the theoretical curve (marked in black). However, the absorption region does not appear show any trend of decrease in $\mathrm{Ly} \alpha / \mathrm{H} \alpha$ ratio with $E_{B-V}$. Instead, we observe a dispersion, as seen in the Ly $\alpha-E_{B-V}$ plot, ranging from $E_{B-V} \sim 0$ to 0.5 .

\subsection{Emission systems: NGC 6090 and IRAS 08339+6517}

Both of these galaxies exhibit Ly $\alpha$ only in emission, with little signs of direct absorption, as seen in Figs. 1 and 2. As usual, the first plot in Figs. 6 and 7 shows Ly $\alpha$ flux function of the color excess $E_{B-V}$. In both systems, we observe that the diffuse component is independent of $E_{B-V}$ at low Ly $\alpha$ flux. Although the calibration procedure used for IRAS $08339+6517$ data is not accurate (see Sect. 2.2), the average extinction found for this galaxy $\left(E_{B-V} \sim 0.12\right)$ is close to the value estimated by Gonzalez Delgado et al. (1998). STIS spectroscopy (Mas-Hesse et al. 2003) revealed a P Cygni profile, with a red wing shifted by $\sim 300 \mathrm{~km} \mathrm{~s}^{-1}$ with respect to the HII region velocity determined from the optical emission lines. The extension of the neutral gas shell is found to be large with a diameter of around $10 \mathrm{kpc}$, which is larger than the aperture used here to isolate the emission region in the center of the galaxy $(\sim 2 \mathrm{kpc})$. Thus, the central emission (red component) is observed through an expanding shell approaching us at a velocity of $\sim 300 \mathrm{~km} \mathrm{~s}^{-1}$. Same Ly $\alpha$ profile has been observed for NGC 6090 by Gonzalez Delgado et al. (1998), with a velocity offset with respect to blueshifted interstellar absorption lines, indicative of large scale high-velocity outflows of gas, of several hundred $\mathrm{km} \mathrm{s}^{-1}$. We differentiate two emission blobs (red for A and green for B in Fig. 7). Component A appears at a mean $E(B-V)$ around 0.74 whereas the second one at $E(B-V) \sim 0.54$. The diffuse emission, also in this galaxy, is the only component that attains the recombination level (Fig. 4), while the bulk of the emission remains below. Again, regarding these two galaxies, the ISM kinematics must play an important role in the escape of Ly $\alpha$ photons through neutral gas shells, making Ly $\alpha$ less sensitive to the dust content. It leads to the observed dispersion in the emission over a large range of extinction for these two galaxies. To confirm the results of this visual inspection, we performed a Spearman's statistical test to measure the probability that a correlation between Ly $\alpha$ and $E_{B-V}$ exists. The Spearman's correlation coefficient $\rho$ can take values from -1 to +1 . A value of +1 shows that the variables are perfectly correlated with a positive slope, a value of -1 indicates that the variables are anti-correlated (negative slope), and a value of 0 implies that the variables are completely independent. While we would expect an anti-correlation between these two variables, we found $\rho \sim 0.15$ for both galaxies (whereas $\rho \sim-0.2$ and -0.05 for Haro 11 and ESO 338-04 respectively), confirming that $\operatorname{Ly} \alpha$ is less sensitive to dust in these systems. For SBS 335-052 we measured $\rho \sim-0.56$.

In IRAS $08339+6517$, the Ly $\alpha$ equivalent width does not appear to be correlated with dust. This absence of correlation is evident from the evolution of the line ratio $\operatorname{Ly} \alpha / \mathrm{H} \alpha$, which is about 2 and remains constant as extinction increases supporting the importance of the ISM kinematics. The situation is slightly different for NGC 6090, where in knot A values are clearly above the theoretical $\operatorname{Ly} \alpha / \mathrm{H} \alpha$ curve (as seen for Haro 11). In addition to kinematics, we may be in presence of clumpy ISM configuration as well (as suggested by the images), that allows Ly $\alpha$ to escape the ionized inter-cloud medium. Furthermore, the two knots show different equivalent widths: $E W_{\mathrm{Ly} \alpha} \sim 54 \AA$ in knot $\mathrm{A}$ and $20 \AA$ in knot $\mathrm{B}$. We note that the diffuse emission has higher values because of the numerous resonant scatterings experienced by these photons. This is also valid for $\mathrm{Ly} \alpha / \mathrm{H} \alpha$ ratio.

\subsection{Damped absorption system: SBS 335-052}

HST/GHRS spectroscopy (Thuan et al. 1997) has revealed that these metal-deficient BCDs are damped Ly $\alpha$ absorbers. In our 

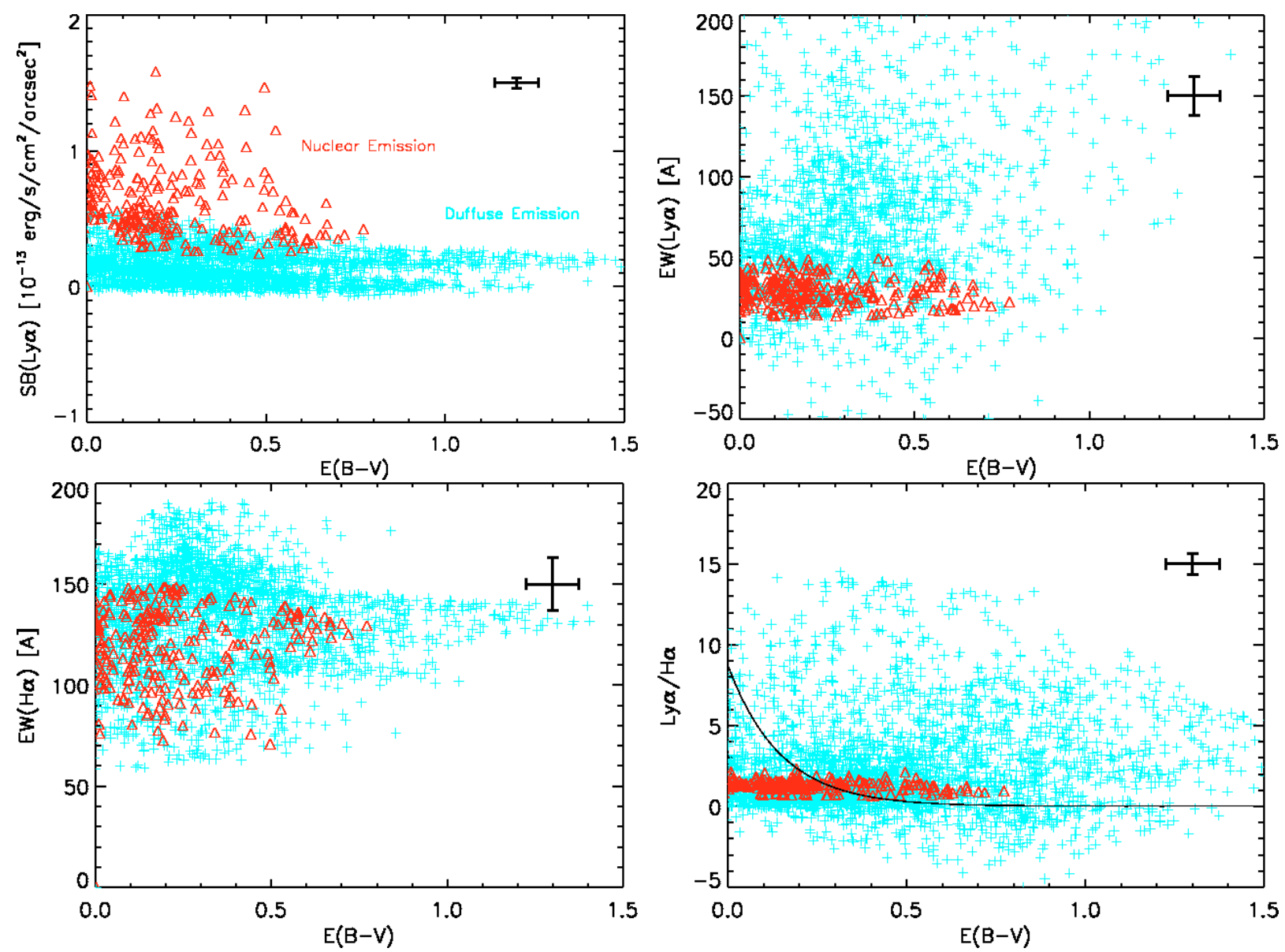

Fig. 6. IRAS $08339+6517$ scatter plots. Top-left: $\operatorname{Ly} \alpha$ surface brightness vs. $E(B-V)$. The nuclear Ly $\alpha$ emission component is represented in red, and the diffuse emission component is in cyan. Same color code applied in all the plots. Top-right: Ly $\alpha$ equivalent width vs. $E(B-V)$. Bottom-left: $\mathrm{H} \alpha$ equivalent width vs. $E(B-V)$. Bottom-right: $\mathrm{Ly} \alpha / \mathrm{H} \alpha$ ratio vs. $E(B-V)$. Error bars correspond to the uncertainties estimated in Sect. 2.3 .

images, they do not show any direct emission but a low surface brightness diffuse halo. The Ly $\alpha$ photons manage to escape from these galaxies after multiple scattering events. Figure 8 shows how Ly $\alpha$ is related to different physical parameters. For $S B S$ 335-052, we see a weak diffuse component in the first frame, which accounts for the entire Ly $\alpha$ emission. Where dust is the main parameter responsible for the destruction of Ly $\alpha$ photons, we expect to measure a negative correlation between Ly $\alpha$ flux and dust extinction. It is precisely what we observe in this galaxy. A significant absorption $\left(f_{\text {Ly } \alpha} \sim-3 \times\right.$ $\left.10^{-12} \mathrm{erg} \mathrm{s}^{-1} \mathrm{~cm}^{-2} \operatorname{arcsec}^{-2}\right)$ is observed, with a weak declining trend in the range $0 \lesssim E_{B-V} \lesssim 0.7$. This result suggests that the dust is playing, in this case, an important role in the escape of Ly $\alpha$ photons. Very Large Array $21 \mathrm{~cm}$ observations (Thuan \& Izotov 1997) indicate that the BCD is embedded in a large HI cloud. The HI column density in the GHRS aperture derived by these authors is large, $N(\mathrm{HI})=7.0 \times 10^{21} \mathrm{~cm}^{-2}$. According to Mas-Hesse et al. (2003) and the evolutionary models of TenorioTagle et al. (1999), this galaxy is a very young starburst of stellar population age that is too small to have ionized the entire surrounding medium, which implies a great amount of neutral gas covering the massive stars. In addition, since the $21 \mathrm{~cm}$ and emission line velocities agree well, the HI cloud is static with respect to the central HII region. Follobing significant scattering by hydrogen atoms, the $\operatorname{Ly} \alpha$ photons increase their mean path and probability of being absorbed by dust grains. The combination of a high HI column density in front of the Ly $\alpha$ production sites and the absence of gas kinematics in this neutral envelope ensures that Ly $\alpha$ photons have a high probability of being destroyed by dust, which implies that dust extinction is an important Ly $\alpha$ escape regulator. This configuration and its related dust correlations were observed for the knot A in Haro 11. It also appears that the Ly $\alpha$ equivalent width is decreases with extinction.

In the same way, TOLOLO 65 shows weak Ly $\alpha$ emission from a diffuse component without any direct emission. For an acceptable signal-to-noise ratio although poorer than that of SBS 335-052, GHRS spectrum (Thuan \& Izotov 1997) shows that this galaxy is a pure Ly $\alpha$ absorber. This is consistent with the very faint and diffuse emission found here, which is not significantly higher than the background level when the degradation in resolution removes the absorption seen in the original HST/ACS image by Östlin et al. (2008).

\section{Discussion}

We discuss the global characteristics of the galaxies studied, based on integrated quantities in clearly defined apertures. This is in contrast to the detailed description of each galaxy presented in the previous section, which enables us to investigate how $\operatorname{Ly} \alpha$ emission is related to other parameters, such as dust, on small scales. For all galaxies, these apertures are defined by masking regions that show $f(\mathrm{H} \beta)$ below a threshold of 5 times the standard deviation of the background in the $\mathrm{H} \beta$ image. Since we study only six galaxies, we are unable of course to obtain 

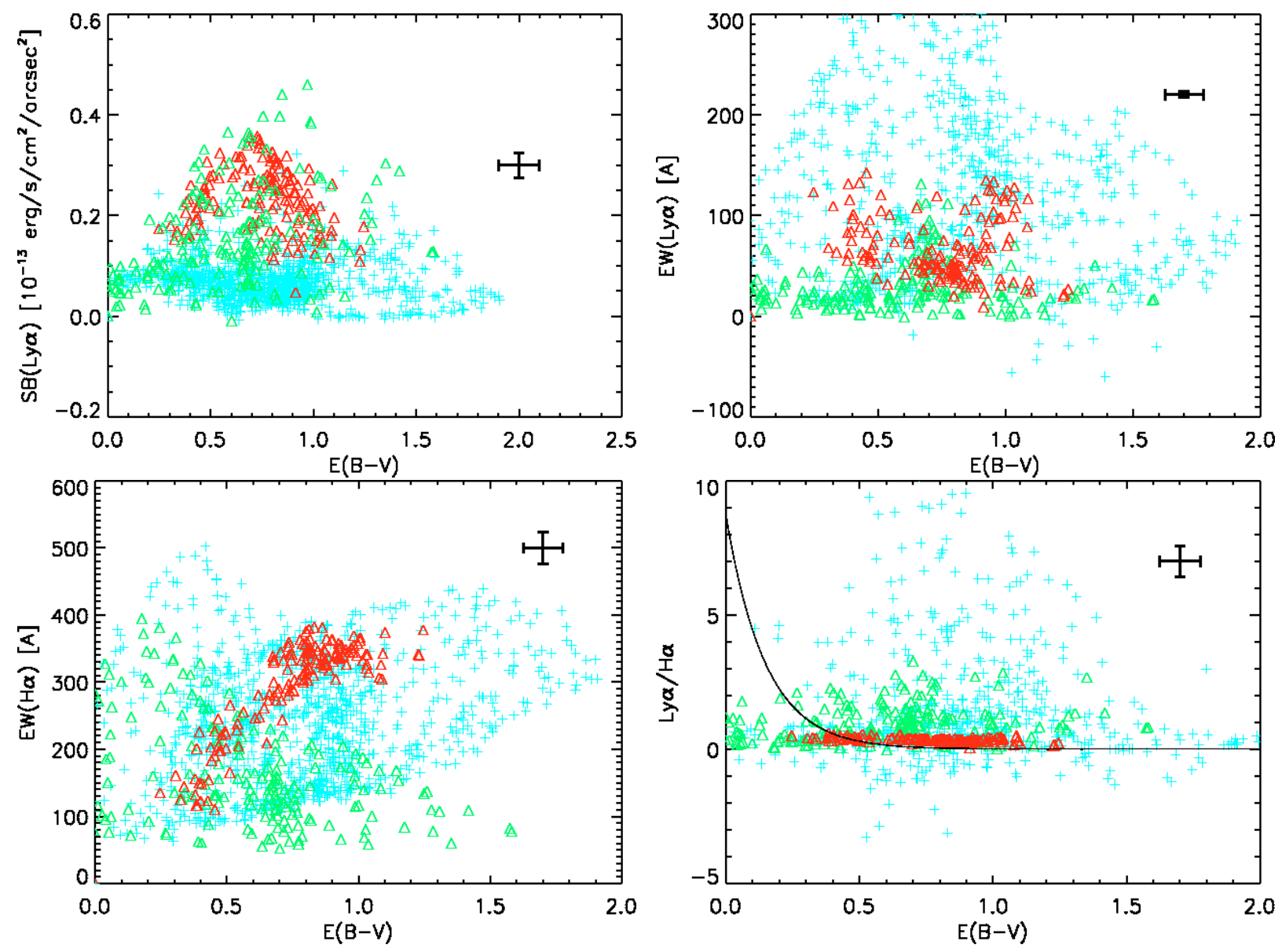

Fig. 7. NGC 6090 scatter plots. Top-left: Ly $\alpha$ surface brightness vs. $E(B-V)$. Emission from knot A is in red, and from knot B is in green. Diffuse emission component is represented as usual in cyan. Same color code applied in all the plots. Top-right: Ly $\alpha$ equivalent width vs. $E(B-V)$. Bottom-left: $\mathrm{H} \alpha$ equivalent width vs. $E(B-V)$. Bottom-right: $\mathrm{Ly} \alpha / \mathrm{H} \alpha$ ratio vs. $E(B-V)$. Error bars correspond to the uncertainties estimated in Sect. 2.3.

statistically meaningful results, even though our galaxies were selected to cover the widest possible range of starburst physical parameters. Nevertheless, we outline some interesting trends in comparison with the small-scale approach and determine how significantly some properties could be smoothed, or not, on the galaxy scale.

\subsection{The role of the dust in Ly $\alpha$ obscuration}

We show in Table 4 the photometric properties of our sample; these represent measurements integrated in an aperture defined by a mask based upon a $\mathrm{H} \beta$ flux threshold above the sky level, which correspond to the extended emission of the Balmer lines and the Ly $\alpha$ diffuse emission. As mentioned before, all quantities presented were corrected for galactic extinction using the methodology of Schlegel et al. (1998) method. By integrating of the Ly $\alpha$ flux within the mask aperture, we detect five candidates in our sample that are emitters and only one net absorber (SBS 335-052). Defining this galaxy as a net absorber implies that the sum of the flux emitted by the entire galaxy corresponds to a negative measurement, although emission is observed in some regions. The flux and the measurements derived are, however, sensitive to the aperture size. We expect that we lose some of the weak diffuse Ly $\alpha$ emission and the most extended $\mathrm{H} \alpha$ or $\mathrm{H} \beta$ emission, which could be attained by deeper observations. Thus, measurements such as the escape fraction of
Ly $\alpha$ may change according to the adopted mask size since Ly $\alpha$ can scatter further from the production sites than Balmer or continuum photons.

The $\mathrm{Ly} \alpha / \mathrm{H} \alpha$ ratio ranges from -1.12 to 1.36 , showing Ly $\alpha$ emission far weaker than predicted by recombination theory even, in most cases, when corrected for the differential extinction at these different wavelengths. Previous observations (Terlevich et al. 1993; Giavalisco et al. 1996) yielded the same conclusions. The dust is just the final stage of the process responsible for the obscuring Ly $\alpha$ photons, after resonant scattering in an homogeneous medium increases their mean path implying that the recombination ratio is not only regulated by the dust (as seen from the $\mathrm{H} \alpha / \mathrm{H} \beta$ ratio, which traces the nebular dust). By searching for correlations between the $\operatorname{Ly} \alpha$ equivalent width and the different parameters of Table 4, we find no clear trends.

Plotting the Ly $\alpha$ equivalent width against $E(B-V)$ allows us to probe the difference in extinction between resonant and nonresonant radiations, with the knowledge that $E W_{\mathrm{Ly} \alpha}$ is unaffected by selective extinction (i.e. independent of the dust extinction curve). We see, indeed, in Fig. 9, a rather scattered set of data points and no well-observed correlation. Since we are dealing with resonant radiation investigated from only one line-of-sight, we expect that the geometry and the distribution of the dust layers around the emitting regions may affect the observed scatter of $E W_{\mathrm{Ly} \alpha}$ according to the extinction. This relation depends also on the intrinsic $E W \mathrm{~s}$. 

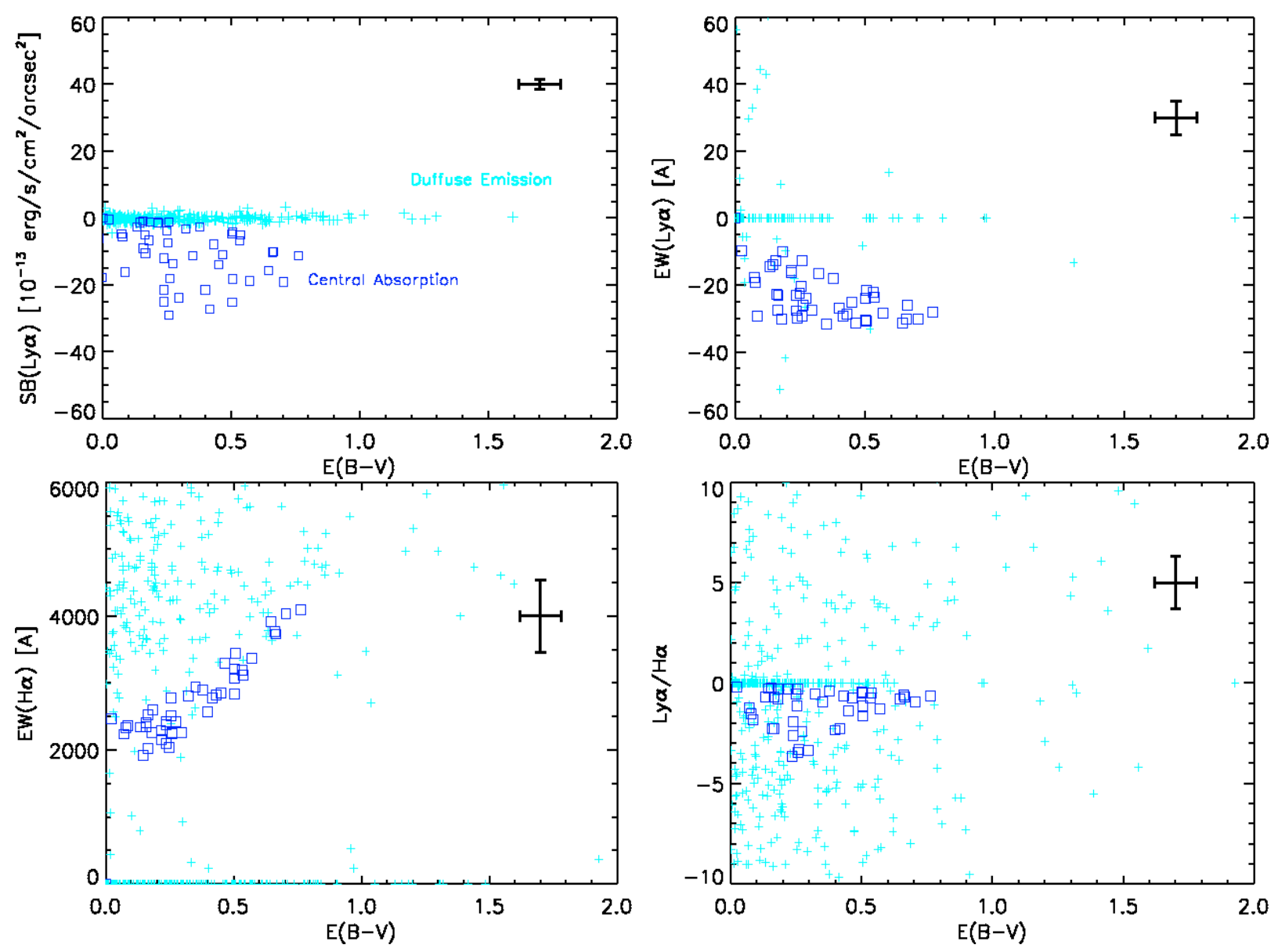

Fig. 8. SBS 335-052 scatter plots. Top-left: Ly $\alpha$ surface brightness vs. $E(B-V)$. The damped central absorption is represented in red, and the diffuse emission component is in cyan. Same color code applied in all the plots. Top-right: Ly $\alpha$ equivalent width vs. E(B - V). Bottom-left: H $\alpha$ equivalent width vs. $E(B-V)$. Bottom-right: $\mathrm{Ly} \alpha / \mathrm{H} \alpha$ ratio vs. $E(B-V)$. Error bars correspond to the uncertainties described in Sect. 2.3.

Table 4. Integrated fluxes and equivalent widths for the six galaxies in the sample. The integration aperture is defined by masking regions below a certain threshold based upon $\mathrm{H} \beta$ flux $\left(f_{\mathrm{H} \beta} \geq 5 \sigma\right.$, where $\sigma$ is the background standard deviation in the same line). The aperture size is given in the first column in $(")^{2}$ and in $\mathrm{kpc}^{2}$ in parentheses. The quantities are corrected for galactic extinction (Schlegel et al. 1998) but not for internal reddening, except for $(\mathrm{Ly} \alpha / \mathrm{H} \alpha)_{\mathrm{C}}$, which has been dereddened with $E_{B-V, \mathrm{gas}}$ using Cardelli et al. (1989) parameterization.

\begin{tabular}{|c|c|c|c|c|c|c|c|c|c|}
\hline Target & $\begin{array}{l}\text { Aperture } \\
\text { size }\end{array}$ & $\begin{array}{c}f_{\mathrm{Ly} \alpha} \\
\left(\mathrm{erg} \mathrm{s}^{-1} \mathrm{~cm}^{-2}\right)\end{array}$ & $\begin{array}{c}f_{\mathrm{H} \alpha} \\
\left(\mathrm{erg} \mathrm{s}^{-1} \mathrm{~cm}^{-2}\right)\end{array}$ & $\begin{array}{c}f_{\mathrm{H} \beta} \\
\left(\mathrm{erg} \mathrm{s}^{-1} \mathrm{~cm}^{-2}\right)\end{array}$ & Ly $\alpha / \mathrm{H} \alpha$ & $(\operatorname{Ly} \alpha / \mathrm{H} \alpha)_{\mathrm{C}}$ & $\mathrm{H} \alpha / \mathrm{H} \beta$ & $\begin{array}{c}E W(\operatorname{Ly} \alpha) \\
(\AA)\end{array}$ & $\begin{array}{c}E W(\mathrm{H} \alpha) \\
(\AA)\end{array}$ \\
\hline Haro 11 & $143(23)$ & $1.3 \mathrm{e}-12$ & $2.3 \mathrm{e}-12$ & $5.48 \mathrm{e}-13$ & 0.57 & 5.48 & 4.14 & 22.8 & 523 \\
\hline ESO 338-IG04 & $267(10)$ & $2.5 \mathrm{e}-12$ & $2.5 \mathrm{e}-12$ & $8.1 \mathrm{e}-13$ & 0.98 & 1.69 & 3.12 & 15.8 & 479 \\
\hline SBS 0335-052 & $3.40(0.23)$ & $-4.0 \mathrm{e}-13$ & $3.6 \mathrm{e}-13$ & $9.9 \mathrm{e}-14$ & -1.12 & -4.8 & 3.62 & -27 & 808 \\
\hline NGC 6090 & $147(49)$ & $6.6 e-13$ & $1.4 \mathrm{e}-12$ & $2.1 \mathrm{e}-13$ & 0.46 & 82 & 6.66 & 62 & 180 \\
\hline IRAS 08339+6517 & $157(23)$ & $3.1 \mathrm{e}-12$ & $2.3 e-13$ & $6.9 \mathrm{e}-13$ & 1.36 & 3 & 3.26 & 45.6 & 140 \\
\hline Tololo 65 & $22.7(0.75)$ & 5.e-14 & $1.8 \mathrm{e}-13$ & $4.9 \mathrm{e}-14$ & 0.28 & 1.15 & 3.6 & 9.1 & 1153 \\
\hline
\end{tabular}

When we calculate the dereddened ratio $(\operatorname{Ly} \alpha / \mathrm{H} \alpha)_{\mathrm{C}}$, rather than $E W_{\mathrm{Ly} \alpha}$, in Table 4, we observe that it is below the recombination value (8.7), which is expected if the extinction is only due to dust, for five galaxies. Only NGC 6090 has a measured value of $(\mathrm{Ly} \alpha / \mathrm{H} \alpha)_{\mathrm{C}}$ that exceeds this recombination level. Since the resonant $\mathrm{Ly} \alpha$ radiation is spatially decoupled from continuum or Balmer lines, the Ly $\alpha$ photons may experience different extinction than traced by the Balmer decrement and lead to this overestimation of the reddening correction. In other words, the detected Ly $\alpha$ photons that have survived numerous scattering and extinction events, have travelled through ISM regions where the amount of dust departs locally from the average. Including a large part of the Ly $\alpha$ diffuse emission in the integration aperture could, for the same reason, contribute to this effect.

We retain from the above analysis that the assumption of simple dust extinction correction fails to recover the intrinsic Ly $\alpha / \mathrm{H} \alpha$ ratio, where the role of the dust is, in most cases, underestimated because of the resonant scattering phenomenon of $\operatorname{Ly} \alpha$.

\subsection{Galaxy sample and Lyo emission morphology}

We present a comparison of our sample properties with those of their high redshift counterparts, such as Lyman Break 


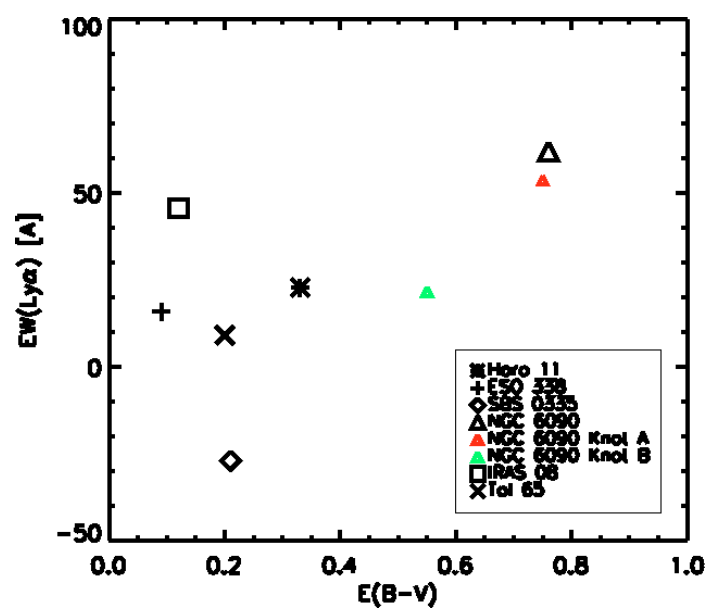

Fig. 9. Scatter plot of integrated quantities, representing in each galaxy the $\operatorname{Ly} \alpha$ equivalent width as a function of the extinction. $E(B-V)$ is derived from the ratio between the integrated $\mathrm{H} \alpha$ and $\mathrm{H} \beta$ fluxes. The legend of symbols assigned to the galaxies are showed in the inset. We have included in this plot the two components of NGC 6090 that we have considered separately.

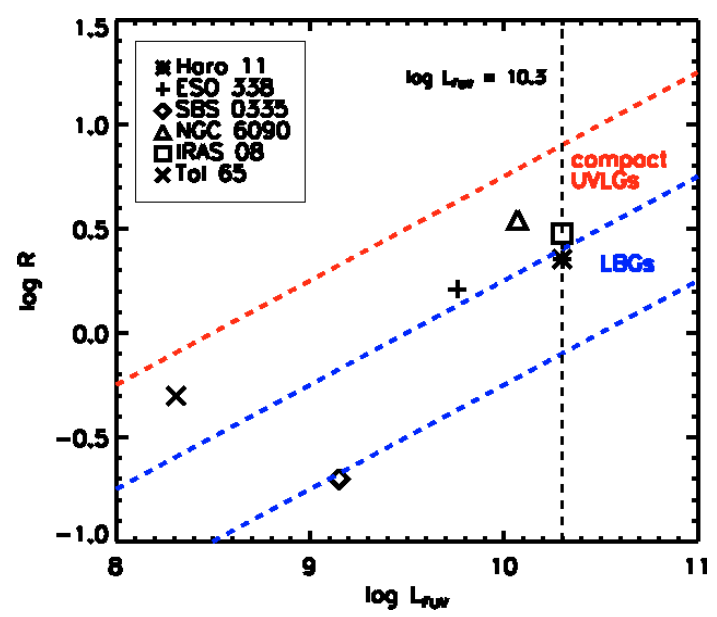

Fig. 10. FUV luminosity derived from F140LP flux (1500 ̊) in units of $L_{\odot}$ versus galaxy radius (in kpc). The masking technique based on the FUV background is applied to derive integrated $L_{\mathrm{FUV}}$ and the equivalent radius $R_{\mathrm{UV}}=\sqrt{\operatorname{area} / \pi}$. The upper dotted line corresponds to compactlarge UVLGs threshold and lower ones are LBG limits in terms of $l_{\mathrm{FUV}}$ (see text). LBGS and UVLGs spaces are marked on the figure.

Galaxies (LBGs). The FUV luminosity is that derived by integrating within the apertures based on FUV background mask and the radius corresponds to $R_{\mathrm{UV}}=\sqrt{\mathrm{area} / \pi}$. The galaxies span a large range in FUV luminosity $8.3 \leq \log \left(L_{\mathrm{FUV}} / L_{\odot}\right) \leq 10.3$ that reaches, for two galaxies, the characteristic LBGs luminosity, and are relatively compact systems, similar to LBGs radii range $\left(\log \left(R_{\mathrm{UV}}(\mathrm{kpc})\right) \sim 0-0.5\right)$. Accordingly, the FUV surface brightness $l_{\mathrm{FUV}}$ of the sample corresponds to that observed in Ultraviolet Luminous Galaxies (UVLGs), as defined by Heckman et al. (2005), with $l_{\mathrm{FUV}} \geq 10^{8} L_{\odot} \mathrm{kpc}^{-2}$ that classifies them in the "compact" category. Figure 10 illustrates the compacity of our galaxies among the UVLGs and LBGS, with their respective classification criteria on $L_{\mathrm{FUV}}$ and $l_{\mathrm{FUV}}$ overplotted. With reference to discussions about local objects and the implications for high- $z$ observations discussed later, we note that Haro 11 and IRAS $08339+6517$, could, according to their SFR and metallicity, be considered to be UVLGs and LBG analogs.
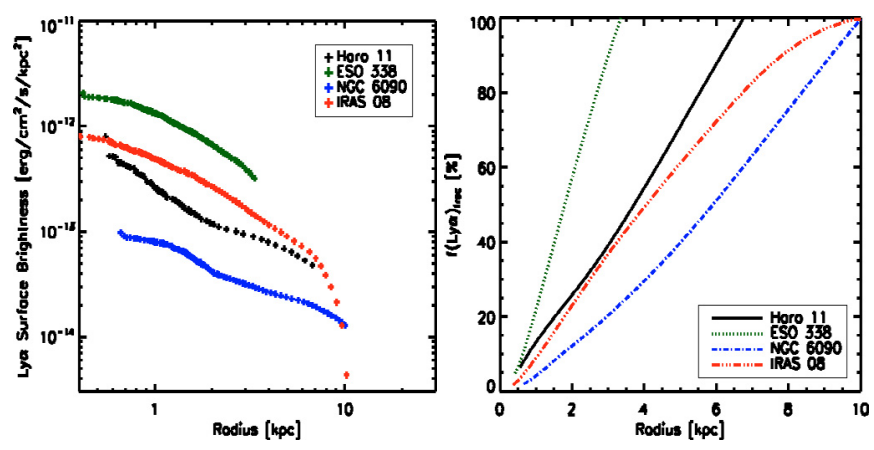

Fig. 11. Ly $\alpha$ emission morphology. The left plot shows the Ly $\alpha$ surface brightness integrated in radius bins against the distance from the brightest emission center. On the right plot, we see the evolution of the Ly $\alpha$ emission fraction cumulated in varying apertures, as a function of the equivalent radius of these apertures. It shows what fraction of Ly $\alpha$ is emitted within a given aperture around the bright emission center. SBS 335-052 and Tololo 65, which show no direct bright but only weak diffuse emission, are not represented.

One of the consequences of the decoupling of $\operatorname{Ly} \alpha$ from nonresonant radiation is the diffuse emission halo observed in all our sample. We present in Fig. 11 the fraction of this diffuse component with respect to the total Ly $\alpha$ flux as a function of the equivalent radius from the brightest emission source which has been isolated in each galaxy to produce the scatter plots of Sect. 3 . The radius is derived from the surface size covered by the emission. SBS 335-052 and Tololo 65 are not represented in the figure since they do not show any bright (direct) emission that might be identified as photon production sources and are only dominated by weak diffuse emission. At a distance of $2 \mathrm{kpc}$, the Ly $\alpha$ emission reaches $40 \%$ of the total galaxy emission in only one galaxy, and for the remaining galaxies, it is still around $20 \%$ or below. We need to integrate until 3-10 kpc (depending on galaxies) to account for the complete Ly $\alpha$ flux. These considerations are relative to the threshold level reached by the observations. Because of the masking technique, we consider that above $80 \%$ of the total emission we are background limited. However, we can infer from these plots that the bulk of $\operatorname{Ly} \alpha$ emission originates in a low surface brightness region.

The situation is slightly different for galaxies not plotted in which the decrease of the surface brightness with radius is less steep for reasons stated above. This illustrates the domination of the photon diffusion mechanism in the escape of Ly $\alpha$ photons, which appears at low surface brightness and extends to large physical scales (several kpc).

\subsection{Age and evolutionary effects}

We computed the mean age of each galaxy from the SED fitting output in each resolution element with two stellar components as free parameters assuming in both of them an instantaneous burst, and averaging over the entire integration aperture by weighting the age in each resolution element by the corresponding $\mathrm{H} \alpha$ luminosity:

mean age $=\frac{\sum_{i} L_{\mathrm{H} \alpha, i} \times \mathrm{age}_{i}}{\sum_{i} L_{\mathrm{H} \alpha, i}}$

apart from Tololo 65, for which no HST H $\alpha$ image is available and the luminosity in the $B$ band is used for weighting. We also completed Monte Carlo simulations to estimate the errors in the weighted age. Each pixel was resampled with a set of 1000 data points and the fitting procedure was applied to this new sample. 

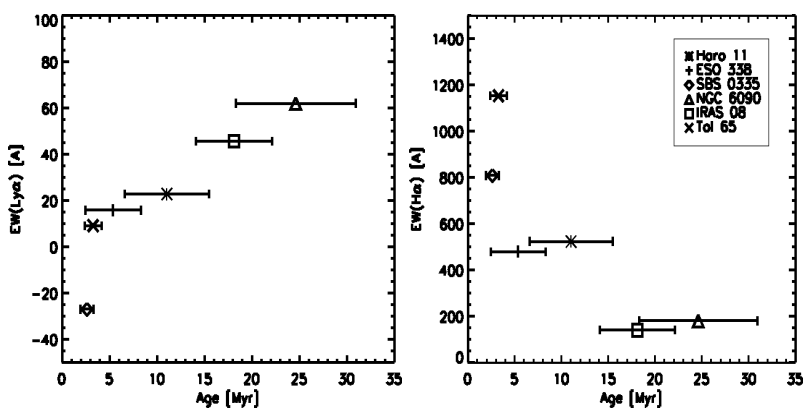

Fig. 12. Age correlations: the left plot shows the integrated $E W_{\mathrm{Ly} \alpha}$ against the mean age integrated over the galaxy. On the right, $E W_{\mathrm{H} \alpha}$ is plotted as a function of the age. 1- $\sigma$ errors on the age fit are also shown. See text for age calculation and errors estimate. Each point represents a galaxy and the legend is given in the inset.

The standard deviation corresponds to the $1-\sigma$ errors plotted in Fig. 12.

The equivalent widths of strong hydrogen recombination lines are known to be, in principle, good age indicators since they measure the ratio between young ionizing over old non-ionizing radiations (Leitherer 2005, and references therein). In Fig. 12, we observe an anticorrelation between $\mathrm{H} \alpha$ equivalent width and the age of the galaxy. This is what is expected from SED models (Starburst 99 for instance) for both $\mathrm{H} \alpha$ and $\mathrm{Ly} \alpha$ equivalent widths and ages $\geq 1 \mathrm{Myr}$ and can be attributed to the decrease in number of ionizing photons quantity and increase in number of stars that contribute to increase in the continuum with time. However, the dispersion observed reflects the complexity of this indicator in practice. Among other effects, $E W_{\mathrm{H} \alpha}$ can be affected by the difference in reddening between nebular and continuum radiations and also radiation from underlying an older stellar population diluting the light of the continuum.

By considering the Ly $\alpha$ equivalent width, we derive a different result. WE are unable to find that $E W_{\mathrm{Ly} \alpha}$ follows the evolutionary sequence in which an increase is observed as a function of the age. The present observations show the additional complexity of $\operatorname{Ly} \alpha$ since we are comparing in the same plot different galaxies affected by different mechanisms, such as expanding shells or static media, and/or probably clumpy ISM, which explains the general difficulty in interpreting the measurement of Ly $\alpha$. Large variations in the observed $E W_{\mathrm{Ly} \alpha}$ are measured for LBGs which have almost identical intrinsic $E W_{\mathrm{Ly} \alpha}$. Schaerer \& Verhamme (2008) showed that, for an extinction of $E(B-V) \sim 0.3$, an intrinsic emission with $E W_{\mathrm{Ly} \alpha} \geq 60 \AA$ is transformed into an absorption, and hence a negative measurement of $E W_{\mathrm{Ly} \alpha}$. Radiation transfer and dust effects can lead to large differences between intrinsic and observed Ly $\alpha$ equivalent widths. In addition, the appearance and evolution of superwinds is a function of age, and we expect that $E W_{\mathrm{Ly} \alpha}$ rises in the presence of ISM kinematical effects. Because of the limited size of the data set that we analyze, this could be considered only as a first step toward a more detailed and significant investigation. It is however worth noting that the small age ( 2.6 Myr) of SBS 335-052 agrees with our discussion about this galaxy as a young starburst embedded in a static HI cloud, which produces a damped absorption. This is also true for Tololo 65 ( $3.3 \mathrm{Myr})$ where no direct emission is seen and for which damped absorption is detected in GHRS spectrum.

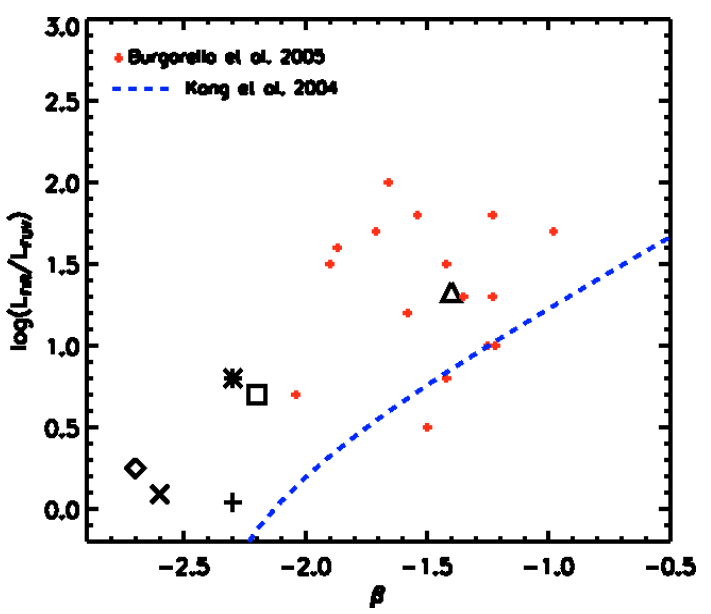

Fig. 13. $L_{\mathrm{IR}}$ to $L_{\mathrm{FUV}}$ ratio versus the UV slope $\beta$. Our galaxies are plotted with the same symbols as in previous figures. $\beta$ is determined from the SED fitting. The remaining red points are galaxies from ELAIS S1 observations in Burgarella et al. (2005). The law describing the relationship between FIR to FUV luminosity ratio and the slope $\beta$, deduced from UV observations (Kong et al. 2004) is overplotted (dashed blue line).

\subsection{Reddening correction and star formation rate}

By studying the evolution in the $L_{\mathrm{FIR}} / L_{\mathrm{FUV}}$ ratio, we develop an alternative way of evaluating the reddening estimate reliability. In Fig. 13, we observe a weak correlation between this luminosity ratio and the UV continuum slope derived from the SED fit. We plotted on the same figure (in blue) the predicted relationship (Kong et al. 2004) between $L_{\mathrm{FIR}} / L_{\mathrm{FUV}}$ and $\beta$, following Meurer et al. (1999). Our galaxies have the same dispersion behavior as those of Burgarella et al. (2005), overplotted as red points, which are more dusty and luminous in the IR. The data point for NGC 6090 is close to the red points because it is classified as a LIRG and is a very dusty starburst with $E_{B-V \text {,gas }} \sim 0.75$. Three of our galaxies (NGC 6090, Haro 11, and IRAS 08339+6517 ) appear to be Luminous Infrared Galaxies (LIRGs, $\left.\log \left(L_{\mathrm{FIR}}\right)>11 L_{\odot}\right)$. In contrast to the IUE starburst sample of Meurer et al. (1999), Goldader et al. (2002) found, as for our galaxies, that the data for LIRGs and ULIRGs of their sample are above the line. Using a large sample of galaxy types, Seibert et al. (2005) derived a correction that lowers the empirical $L_{\mathrm{FIR}} / L_{\mathrm{FUV}}-\beta$ reddening relation and is in disagreement with our observations. The observed discrepancy suggests that a simple empirical law is not representative of the observations and the galaxies experience a higher attenuation than suggested by $\beta$.

Star-formation rate is an essential diagnostic tool of the evolution of galaxies. Since the high-redshift universe has become accessible observationally, star formation episodes and their evolution can be studied over a wide range of epochs (e.g. Madau et al. 1996; Giavalisco et al. 2004). Many indicators employing radiation from rest-frame UV to infrared are used to estimate the SFR. A commonly used toll for studying the distant universe is based on Ly $\alpha$ emission. One of the most critical issues related to estimating the SFR, from UV or optical indicators, is the correction for internal reddening. We need to estimate accurately the dust obscuration and hence the intrinsic flux to complete a correct conversion to star-formation rate. This issue is even more critical for the indicator based on $\operatorname{Ly} \alpha$ emission, considering the radiative transfer complexity of this line discussed in this paper. Table 5 summarizes the SFRs computed from different indicators using Kennicutt (1998) calibrations. The conversion 
Table 5. Star formation rates and luminosities. $L_{\mathrm{FUV}}$ is calculated with $\lambda \times f_{\lambda}$ where $\lambda=1525 \AA$. $f_{\lambda}$ is the flux density in $F 140 L P$ filter. $L_{\mathrm{FIR}}$ is from Östlin et al. (2008). Both are expressed in units of $L_{\odot, B o l}\left(3.8 \times 10^{33} \mathrm{erg} \mathrm{s}^{-1}\right)$. Star formation rates are derived from the integrated fluxes over apertures based on sky background threshold and using the calibration of Kennicutt (1998) (presented in units of $M_{\odot} \mathrm{yr}^{-1}$ ). All quantities are corrected for galactic foreground extinction. The last column ratio only is corrected for internal reddening using $E_{B-V, \text { stars }}$ for SFR ${ }_{\mathrm{UV}}$ correction and $E_{B-V, \mathrm{gas}}$ for $\mathrm{SFR}_{\mathrm{Ly} \alpha}$ one.

\begin{tabular}{lcccccccc}
\hline \hline Target & $\log \left(L_{\mathrm{FUV}}\right)$ & $\log \left(L_{\mathrm{FIR}}\right)$ & $S F R(\mathrm{UV})$ & $S F R(\mathrm{Ly} \alpha)$ & $S F R(\mathrm{FIR})$ & $S F R(\mathrm{H} \alpha)$ & $\left(S F R_{\mathrm{UV}} / S F R_{\mathrm{Ly} \alpha}\right)$ & $\left(S F R_{\mathrm{UV}} / S F R_{\mathrm{Ly} \alpha}\right)_{\mathrm{COR}}$ \\
\hline Haro 11 & 10.3 & 11.1 & 5.1 & 1.07 & 21.7 & 16.33 & 4.77 & 0.53 \\
ESO 338-IG04 & 9.76 & 9.8 & 1.56 & 0.3 & 1.1 & 3.9 & 5.20 & $\ldots$ \\
SBS 0335-052 & 9.1 & 9.4 & 0.34 & $\ldots$ & 0.4 & 1.1 & $\ldots$ & $\ldots$ \\
NGC 6090 & 10.03 & 11.4 & 2.9 & 1.1 & 43.3 & 20.7 & 2.64 & 0.015 \\
IRAS 08339+6517 & 10.3 & 11.0 & 4.4 & 2.2 & 17.3 & 14 & 2 & 0.85 \\
Tololo 65 & 8.3 & 8.4 & 0.05 & 0.008 & 0.05 & 0.24 & 6.25 & 2.6 \\
\hline
\end{tabular}

from flux to SFR using these calibrations assumes a continuous star formation regime in the equilibrium phase, whereas in our SED fitting procedure, used for the Ly $\alpha$ continuum subtraction or age estimation, we have assumed an instantaneous burst scenario. Nevertheless, translating our results into star-formation rates provides a useful comparison with previous works using these widely used calibrations.

UV emission from galaxies traces the young stellar population and a conversion from UV luminosity to SFR can be computed across the UV range (1250-2500 $\AA$ ). This estimation is very sensitive to dust attenuation, because of the wavelength domain and the patchy ISM (Kennicutt 1998) ${ }^{1}$. Alternatively, nebular emission lines, such as $\operatorname{Ly} \alpha$, provide information on the ionizing flux of the young massive stellar population with lifetimes <20 Myr. Therefore, it provides a quasi-instantaneous estimation of the current star-formation rate. For case B recombination theory (Brocklehurst 1971), we have:

$\operatorname{SFR}_{\mathrm{Ly} \alpha}\left(M_{\odot} \mathrm{yr}^{-1}\right)=9.1 \times 10^{-43} L(\mathrm{Ly} \alpha)\left(\mathrm{erg} \mathrm{s}^{-1}\right)$.

In addition to the dependence on the IMF, this method is highly sensitive to the extinction correction as we have mentioned before. Figure 14 is illustrative of the reddening correction issue. Figure 14a presents $\operatorname{SFR}(\operatorname{Ly} \alpha)$ versus SFR(UV): in the case of data shown by the dark points, neither value of SFR is corrected, and for the red points, $\operatorname{SFR}(\operatorname{Ly} \alpha)$ is corrected using $E_{B-V \text {,gas }}$ and

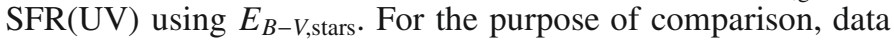
from the literature are also included. It is interesting to note the consistent discrepancy between $\operatorname{SFR}(\operatorname{Ly} \alpha)$ and SFR(UV) (dark points) for both our sample and objects from literature, including low- (Giavalisco et al. 1996) and high-redshift galaxies (Taniguchi et al. 2005; Ajiki et al. 2003; Tapken et al. 2007). The lack of points under the line of equality at low SFR(Ly $\alpha)$ for high- $z$ observations is a consequence of the completeness limit for LAEs in particular, excluding faint Ly $\alpha$ emitters. Due to the resonance effects of $\operatorname{Ly} \alpha$, we expect to observe a scattered distribution below the line of slope unity, provided that a statistically significant sample is used. Such a distribution would help to characterize a potential upper limit to the disagreement between resonant and non-resonant SFR indicators. Such a diagram could also serve as an important probe of galaxy evolution from damped and/or young systems for which $S F R_{\mathrm{Ly} \alpha} \sim 0$, to more evolved starburst events with higher ionized gas fraction and/or undergoing feedback outflows, and therefore approaching the line for which $S F R_{\mathrm{Ly} \alpha} \sim S F R_{\mathrm{UV}}$.

In the case of observed values, for our galaxies $\operatorname{SFR}(\operatorname{Ly} \alpha)$ is systematically below the equal value line, underestimating SFR by a factor of between 2 to 6 with respect to SFR(UV).

${ }^{1} \operatorname{SFR}_{\mathrm{UV}}\left(M_{\odot} \mathrm{yr}^{-1}\right)=1.4 \times 10^{-28} L_{v}\left(\mathrm{erg} \mathrm{s}^{-1} \mathrm{~Hz}^{-1}\right)$.
Observed usually in high- $z$ galaxies, this discrepancy emphasizes the highest attenuation of the $\operatorname{Ly} \alpha$ emission line with respect to the UV continuum. Correcting for dust attenuation, a principle reason for the discrepancy, ensures that the two star formation rates agree more closely, apart from data for NGC 6090.

When SFR derived from the rest frame UV light is not corrected for absorption, the SFR measured from the infrared should, in principle, be complementary, since radiation that is strongly absorbed in the UV is re-emitted in the thermal $\mathrm{IR}^{2}$.

Figure $14 \mathrm{~b}$ shows the total $\mathrm{SFR}\left(\mathrm{SFR}_{\mathrm{UV}}+\mathrm{SFR}_{\mathrm{FIR}}\right)$ where $\mathrm{SFR}_{\mathrm{UV}}$ is not corrected, versus dereddened $\mathrm{SFR}_{\mathrm{Ly} \alpha}$ and $\mathrm{SFR}_{\mathrm{H} \alpha}$ with $E_{B-V \text {,gas }}$ (dark and blue points), and SFR $\mathrm{UV}_{\mathrm{UV}}$ with $E_{B-V \text {,stars }}$ (red points). The corrected $\operatorname{SFR}(\operatorname{Ly} \alpha)$ remains below the total SFR for most galaxies apart from NGC 6090 for which the dust correction highly overestimates the total SFR (we have discussed possible reasons for this overestimation in Sect. 4.1). In contrast, SFR measurements derived from corrected $\mathrm{H} \alpha$ (blue points) luminosity are closer in value to the true measurements. Similarly, the dereddened UV estimator (red points) places the galaxy data points rather close to the line of equality. Differences in SFR measurements derived using $\operatorname{Ly} \alpha$ radiation and other indicators such as $\mathrm{H} \alpha$ or $\mathrm{UV}$ and the failure of the $\mathrm{Ly} \alpha$ indicator to recover the total SFR (UV + IR) even after correcting for reddening, are the result of the decoupling of resonant $\operatorname{Ly} \alpha$ and non resonant (e.g. UV continuum or Balmer lines) radiation with respect to the dust obscuration, which explains the difficulty in using this line as a reliable star formation indicator.

As an alternative, we take advantage of the available information from our observations to improve our estimate of the star formation rate when only Ly $\alpha$ luminosity is known. We calculate the Ly $\alpha$ escape fraction using the corrected $\mathrm{H} \alpha$ flux and assuming the case B recombination ratio $\operatorname{Ly} \alpha / \mathrm{H} \alpha$ :

$$
\begin{aligned}
f_{\mathrm{esc}}(\mathrm{Ly} \alpha) & =f(\operatorname{ly} \alpha) /\left(8.7 \times f(\mathrm{H} \alpha)_{\mathrm{C}}\right) \\
f(\mathrm{H} \alpha)_{\mathrm{C}} & \left.=f(\mathrm{H} \alpha) \times 10^{\left(1.048 \times E(B-V)_{\text {gas }}\right.}\right)
\end{aligned}
$$

where $f(\operatorname{ly} \alpha)$ is the observed flux and $f(\mathrm{H} \alpha)_{\mathrm{C}}$ is the $\mathrm{H} \alpha$ flux corrected pixel by pixel for internal reddening using the Cardelli et al. (1989) extinction law. Unlike high-z observations for which only the global UV slope is known, our present study provides accurate extinction data. Therefore, $f_{\text {esc }}$ is a good estimate of the intrinsic Ly $\alpha$ flux, since it takes into account both dust obscuration and resonant scattering mechanism and hence allows us to correct the $\mathrm{SFR}_{\mathrm{Ly} \alpha}$ calibration (Eq. (3)) as follows:

$S F R_{\mathrm{Ly} \alpha}\left(M_{\odot} \mathrm{yr}^{-1}\right)=\left(1 / f_{\mathrm{esc}}\right) \times 9.1 \times 10^{-43} L(\mathrm{Ly} \alpha)\left(\mathrm{erg} \mathrm{s}^{-1}\right)$

The escape fractions obtained for the six galaxies are reported in Table 6. We can assume, in general terms, an escape fraction

\footnotetext{
${ }^{2} \operatorname{SFR}_{\mathrm{FIR}}\left(M_{\odot} \mathrm{yr}^{-1}\right)=4.5 \times 10^{-44} L_{\mathrm{FIR}}\left(\mathrm{erg} \mathrm{s}^{-1}\right)$.
} 


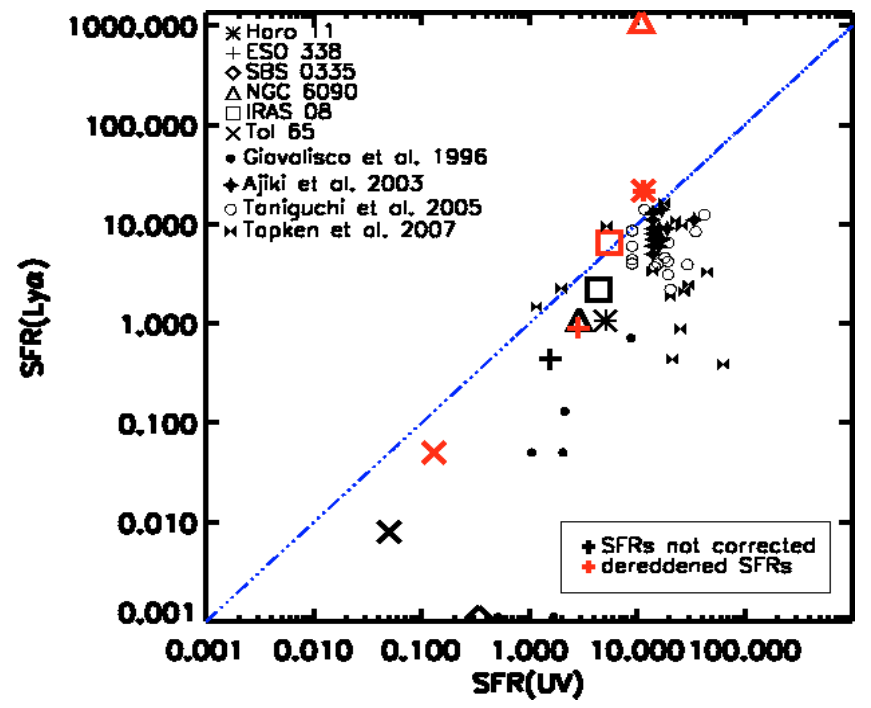

(a) $\mathrm{SFR}_{\mathrm{Ly} \alpha}$ vs. SFR $\mathrm{UV}$

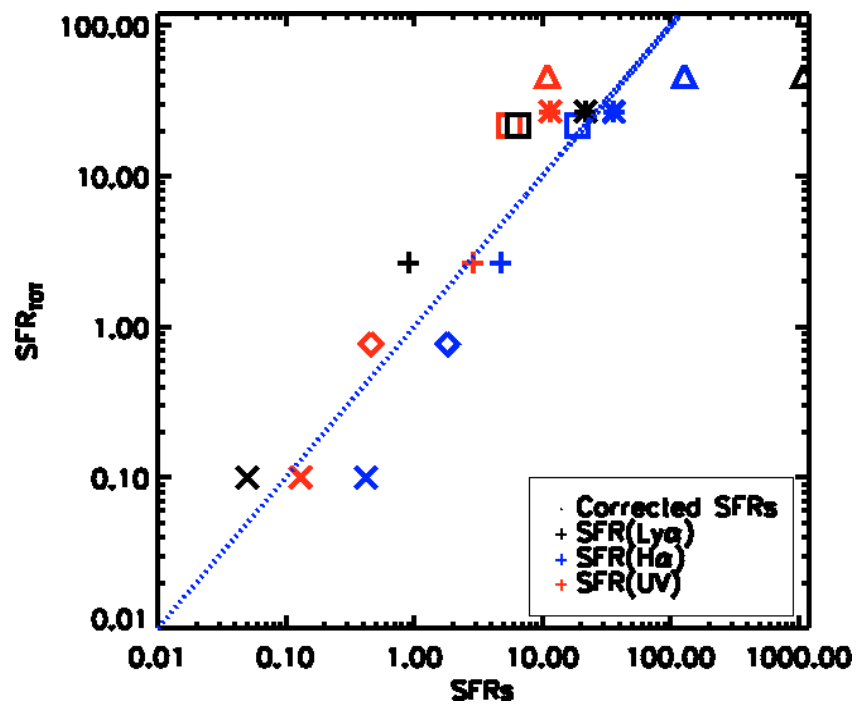

(b) $\mathrm{SFR}_{\mathrm{TOT}}$ vs. different SFRs

Fig. 14. Star formation rates: Fig. 14a shows SFR derived from nebular emission line Ly $\alpha$ versus SFR derived from UV continuum. The dark points represent undereddened SFRs. We note that because of the logarithmic scale and for the sake of readability, we have placed galaxies for which $S F R_{\mathrm{Ly} \alpha}=0$ at $S F R_{\mathrm{Ly} \alpha}=0.001$. Observed SFRs from literature at low-z (Giavalisco et al. 1996, and IUE data from NED) and high- $z$ (Taniguchi et al. 2005; Ajiki et al. 2003, for LAEs) (Tapken et al. 2007, for LBGs) are also overplotted and explained in the legend. For our sample, the red points represent $\mathrm{SFR}_{\mathrm{Ly} \alpha}$ dereddened using $E_{B-V, \mathrm{gas}}$ and $\mathrm{SFR}_{\mathrm{UV}}$ dereddened using $E_{B-V, \text { stars }}$. The dashed line is for $S F R_{\mathrm{Ly} \alpha}=S F R_{\mathrm{UV}}$. Figure $14 \mathrm{~b}$ represents $\mathrm{SFR}(\mathrm{UV}+\mathrm{IR})$ (where $\mathrm{SFR}_{\mathrm{UV}}$ is not corrected for extinction) versus different corrected SFRs. The legend is also marked on the figure. Units are in $M_{\odot} \mathrm{yr}^{-1}$.

Table 6. Integrated properties and $\operatorname{Ly} \alpha$ emission characteristics. quantities are integrated over the same apertures defined in Table 4. The first column contains the escape fraction of $\operatorname{Ly} \alpha$ photons for each galaxy assuming a case B $\mathrm{Ly} \alpha / \mathrm{H} \alpha$ recombination ratio (see text for details). For SBS 0335-052, only positive Ly $\alpha$ contribution is used to derive an upper limit for $f_{\text {esc }}$. Second column represents the contribution of the diffuse component in the whole Ly $\alpha$ emission determined by masking the bright "direct" emission sources if any. The mean nebular extinction $E_{B-V \text {,gas }}$ is derived from the ratio of integrated $\mathrm{H} \alpha$ to $\mathrm{H} \beta$ fluxes and is showed in Col. 3. The fourth gives the continuum extinction determined from the SED fitting procedure. Last column shows the age issued from the SED fitting and averaged on the total galaxy pixels following Eq. (2).

\begin{tabular}{lccccc}
\hline \hline Target & $\begin{array}{c}f_{\text {esc }} \\
(\%)\end{array}$ & $\begin{array}{c}f_{\text {diffuse }} \\
(\%)\end{array}$ & $\begin{array}{c}E(B-V) \\
\text { gas }\end{array}$ & $\begin{array}{c}E(B-V) \\
\text { stars }\end{array}$ & $\begin{array}{c}\text { Age } \\
(\mathrm{Myr})\end{array}$ \\
\hline Haro 11 & 2.6 & 74 & 0.33 & 0.07 & 11 \\
ESO 338-IG04 & 8 & 70 & 0.08 & 0.08 & 5.4 \\
SBS 0335-052 & 0.25 & $\sim 100$ & 0.21 & 0.04 & 2.6 \\
NGC 6090 & 0.56 & 73 & 0.76 & 0.18 & 25 \\
IRAS 08339+6517 & 7 & 65 & 0.12 & 0.03 & 18 \\
Tololo 65 & 1.7 & $\sim 100$ & 0.2 & 0.12 & 3.3 \\
\hline
\end{tabular}

of $5 \%$ as a mean statistical value to deduce the appropriate SFR when $f_{\text {esc }}$ is unavailable. This correction is evidently subject to uncertainties due to $f_{\text {esc }}$ variations, but is in any case more representative of reality than standard calibrations (Kennicutt 1998, for instance).

According to the definition of the escape fraction in Eq. (4), the revised star formation rate based on $L(\operatorname{Ly} \alpha)$ is equivalent to $\mathrm{SFR}(\mathrm{H} \alpha)$ corrected for reddening. Hence, it is represented on the right plot of Fig. 14 by the blue points, which appears to provide a more accurate estimation of the total SFR than that given by the $\mathrm{SFR}_{\mathrm{Ly} \alpha}$ corrected only for dust obscuration.

\subsection{Implications for high-redshift galaxies}

We show in Fig. 15 how $f_{\text {esc }}$ is a function of extinction. We observe that a correlation exists between the Ly $\alpha$ escape fraction and $E_{B-V \text {,gas }}$ but with a significant dispersion in, moreover, a small sample. On the galaxy scale, we observe effects of dust obscuration on the emergent $\operatorname{Ly} \alpha$ radiation; in a purely dustregulated model however, this correlation would be more significant. We note that correcting the total $\mathrm{H} \alpha$ flux using the mean $E_{B-V \text {,gas }}$ produces higher escape fractions by 15 to $40 \%$ than those calculated using pixel level corrections. In high redshift observations, only global corrections are possible when the spatial resolution is poor.

The rate of escaping Ly $\alpha$ photons does not exceed $10 \%$ in the galaxies we study. In addition, we have outlined the ubiquitous halo of diffuse emission present in all observed galaxies, and we determined the importance of the contribution in Sect. 4.2. The diffuse component represents the bulk of the Ly $\alpha$ emission (Table 6). It is probable that such low surface brightness emission remains undetectable in high-redshift galaxies. We note that caution should be taken when deriving physical quantities, such as star formation rates (SFRs), using Ly $\alpha$ line alone, since we are dealing with only a small fraction of escaping photons and probably missing the majority of this fraction. The simple correction of SFR for dust extinction could also be at fault because this method failed to recover the value determined by the recombination theory in most cases and the behavior of resonant radiation, according to the dust content, is unpredictable without any complementary information (HI distribution, gas kinematics, etc.). However a more accurate and realistic estimate could be obtained using the calibration proposed in Eq. (6).

Because of the difficulties discussed above, high- $z$ star formation rates based on Ly $\alpha$ are generally underestimated with respect to those derived for instance from UV. Discrepancies were observed between the two estimation methods, where SFR based on the $\operatorname{Ly} \alpha$ luminosity was smaller by a factor of two or 


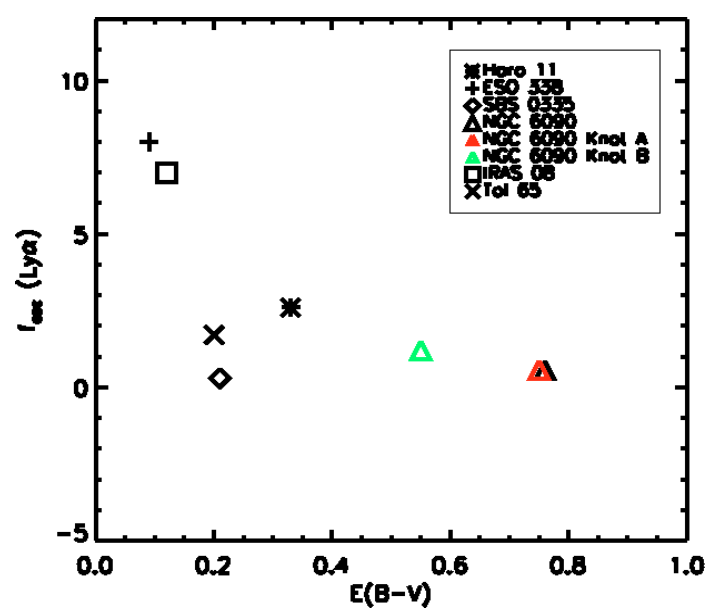

Fig. 15. Escape fraction of $\operatorname{Ly} \alpha$ photons (in percent, see text for details on the $f_{\text {esc }}$ determination) as a function of extinction $E(B-V)$ in the gas phase. We have included the two components (knots A and B) of the interacting system NGC 6090 and treated them separately by a masking procedure. We observe a decline in the amount of escaping photons when increasing the dust amount. We note also that most of the galaxies have a small $f_{\text {esc }}$ around $3 \%$ or below.

more than that based on the UV continuum (Hu et al. 2002; Kodaira et al. 2003). Taniguchi et al. (2005) found that SFRs derived from Ly $\alpha$ for their sample of $z \sim 6.6$ LAEs lie a factor of 5 , on average, below those based on UV continuum. Tapken et al. (2007) found similar discrepancies for their UV-selected galaxies. This is also observed at low redshift, where discrepancy equivalent to a factor of 2 to 6 is found (the present work, Sect. 4.4)

Ly $\alpha$ emission line has became a powerful tracer of star formation at high-redshift. However, it is clear that using only the Ly $\alpha$ luminosity or equivalent width for the characterization of star formation episodes leads to many uncertainties, which are inherent to the Ly $\alpha$ radiative transfer complexity and uncertainties in the SFR calibration methods. Despite differences due to obvious evolution effects between local and high- $z$ star-forming galaxies (see Mas-Hesse et al. 2003), the physical processes governing the radiative transfer and the escape mechanism of Ly $\alpha$ photons should be the same, and support our present use of an extrapolation. Nevertheless, we emphasize again the limited number of galaxies in our sample that precludes any statistically significant study, and propose a further investigation with an extended sample of galaxies and complementary spectroscopic study.

\section{Conclusions}

Combining space (HST) and ground-based (NOT and NTT) observations, we have mapped the Ly $\alpha$ emission and the dust content in six nearby star-forming galaxies. We have compared the extinction $E(B-V)$ produced from the Balmer decrement $\mathrm{H} \alpha / \mathrm{H} \beta$ to several parameters such as $\operatorname{Ly} \alpha$ emission, equivalent width, or recombination ratio $\mathrm{Ly} \alpha / \mathrm{H} \alpha$ on small scales to disentangle the role of the dust from other parameters. Implications for high- $z$ studies inferred from global properties of the galaxies have also been investigated.

Our galaxies exhibit different Ly $\alpha$ morphologies from emission to damped absorption or combination thereof:

- In systems with emission and absorption (namely Haro 11 and ESO 338-04), we found Ly $\alpha$ photons emerging from regions of similar or even higher extinction than those where Ly $\alpha$ is seen in absorption. We point out the role of the ISM distribution, where in the case of clumpyness morphology Ly $\alpha$ photons escape preferentially to $\mathrm{H} \alpha$ ones leading to an observed $\operatorname{Ly} \alpha / \mathrm{H} \alpha$ ratio higher than the theoretical level corrected for extinction.

- In objects that show no strong absorption (NGC 6090 and IRAS $08339+6517)$, we observe no clear correlation between Ly $\alpha$ and the dust content. The HI kinematics may play a more significant role in the escape of Ly $\alpha$ photons as confirmed by kinematics studies, which have shown large ISM outflows in both systems.

- SBS 335-052 is a Ly $\alpha$ absorber with a large Hi column density coverage, which is believed to be static with respect to the emitting region. We estimate an age ( $<5 \mathrm{Myr})$ in agreement with the picture where the starburst is too young to have ionized the surrounding gas or driven an outflow. We observe precisely what is expected from the resonant nature of $\operatorname{Ly} \alpha$ in a static neutral gas: a damped absorption with a declining relationship between $\operatorname{Ly} \alpha$ and $E(B-V)$, indicating that the dust is, in this case, the main regulator of $\operatorname{Ly} \alpha$ escape.

When investigating global parameters of our sample, we found that simple dust extinction correction fails to recover the intrinsic $\mathrm{Ly} \alpha / \mathrm{H} \alpha$ ratio, where the role of the dust is, in some cases, underestimated because of the resonant scattering, and in other cases, overestimated because of the clumpyness distribution of the ISM. We observe neither no evident correlation between $E W_{\mathrm{Ly} \alpha}$ and the reddening. The observed Ly $\alpha$ escape fraction is found not to exceed $10 \%$ in our sample and is, for most of our galaxies, about $3 \%$ or less.

The resonant decoupling of $\operatorname{Ly} \alpha$ from non-resonant radiation leads also to an ubiquitous diffuse halo with low surface brightness. It represents the bulk of the Ly $\alpha$ emission and extend to regions at several $\mathrm{kpc}$ from emitting regions, which cannot be reached by $\mathrm{H} \alpha$ or continuum radiation yielding high $E W_{\mathrm{Ly} \alpha}$.

Because of the radiative transfer complexity of the Ly $\alpha$ line, star formation rates (SFR) measured using Ly $\alpha$ differ from SFRs derived using other indicators (from UV for instance), and fail to recover the total SFR (UV + IR), even when corrected for dust obscuration, preventing any determination of the intrinsic star formation rate. We therefore propose a more realistic calibration of the SFR when information on Ly $\alpha$ only is available (which is usually the case for high-redshift surveys), which accounts for dust attenuation and resonant scattering phenomenon by means of the Ly $\alpha$ escape fraction.

Acknowledgements. We are very grateful to Daniel Schaerer for thoughtful and valuable comments on the draft of this paper. We thank Anne Verhamme for useful discussions, Claus Leitherer and Artashes Petrosian for their work on the Ly $\alpha$ project. Filter 113 for ALFOSC observations was acquired thanks to a grant from Erik Holmberg foundation.

\section{References}

Ahn, S.-H., Lee, H.-W., \& Lee, H. M. 2003, MNRAS, 340, 863 Ajiki, M., Taniguchi, Y., Fujita, S. S., et al. 2003, AJ, 126, 2091 Bergvall, N., \& Östlin, G. 2002, A\&A, 390, 891

Bergvall, N., Zackrisson, E., Andersson, B.-G., et al. 2006, A\&A, 448, 513 Brocklehurst, M. 1971, MNRAS, 153, 471

Burgarella, D., Buat, V., Small, T., et al. 2005, ApJ, 619, L63

Calzetti, D., \& Kinney, A. L. 1992, ApJ, 399, L39

Calzetti, D., Kinney, A. L., \& Storchi-Bergmann, T. 1994, ApJ, 429, 582

Cardelli, J. A., Clayton, G. C., \& Mathis, J. S. 1989, ApJ, 345, 245

Charlot, S., \& Fall, S. M. 1993, ApJ, 415, 580

Cowie, L. L., \& Hu, E. M. 1998, AJ, 115, 1319 
de Propris, R., Pritchet, C. J., Hartwick, F. D. A., \& Hickson, P. 1993, AJ, 105, 1243

Deharveng, J. M., Joubert, M., \& Kunth, D. 1985, in Star-Forming Dwarf Galaxies and Related Objects, ed. D. Kunth, T. X. Thuan, \& J. Tran Thanh van, 431

Dekker, H., Delabre, B., \& Dodorico, S. 1986, Presented at the Society of PhotoOptical Instrumentation Engineers (SPIE) Conference, Instrumentation in astronomy VI, Proceedings of the Meeting, Tucson, AZ, Mar. 4-8, Part 1 (A8736376 15-35), Bellingham, WA, Society of Photo-Optical Instrumentation Engineers, ed. D. L. Crawford, 627, 339

Dijkstra, M., Wyithe, J. S. B., \& Haiman, Z. 2007, MNRAS, 379, 253

Djorgovski, S., \& Thompson, D. J. 1992, in The Stellar Populations of Galaxies, ed. B. Barbuy, \& A. Renzini, IAU Symp., 149, 337

D'Odorico, S., Beletic, J. W., Amico, P., et al. 1998, in Presented at the Society of Photo-Optical Instrumentation Engineers (SPIE) Conference, Optical Astronomical Instrumentation, ed. S. D’Odorico, Proc. SPIE, 3355, 507

Finkelstein, S. L., Rhoads, J. E., Malhotra, S., Grogin, N., \& Wang, J. 2007, ArXiv e-prints, 708

Fujita, S. S., Ajiki, M., Shioya, Y., et al. 2003, AJ, 125, 13

Giavalisco, M., Koratkar, A., \& Calzetti, D. 1996, ApJ, 466, 831

Giavalisco, M., Dickinson, M., Ferguson, H. C., et al. 2004, ApJ, 600, L103

Goldader, J. D., Meurer, G., Heckman, T. M., et al. 2002, ApJ, 568, 651

Gonzalez Delgado, R. M., Leitherer, C., Heckman, T., et al. 1998, ApJ, 495, 698

Grimes, J. P., Heckman, T., Strickland, D., et al. 2007, ApJ, 668, 891

Gronwall, C., Ciardullo, R., Hickey, T., et al. 2007, ApJ, 667, 79

Hamana, T., Ouchi, M., Shimasaku, K., Kayo, I., \& Suto, Y. 2004, MNRAS, 347, 813

Hansen, M., \& Peng Oh, S. 2006, New A Rev., 50, 58

Hayes, M., Östlin, G., Mas-Hesse, J. M., et al. 2005, A\&A, 438, 71

Hayes, M., Östlin, G., Atek, H., et al. 2007, MNRAS, 382, 1465

Hayes, M., Östlin, G., Mas-Hesse, J. M., \& Kunth, D. 2008, AJ, submitted

Heckman, T. M., Hoopes, C. G., Seibert, M., et al. 2005, ApJ, 619, L35

Hu, E. M., Cowie, L. L., \& McMahon, R. G. 1998, ApJ, 502, L99

Hu, E. M., Cowie, L. L., McMahon, R. G., et al. 2002, ApJ, 568, L75

Izotov, Y. I., Lipovetsky, V. A., Chaffee, F. H., et al. 1997, ApJ, 476, 698

Izotov, Y. I., Chaffee, F. H., \& Green, R. F. 2001, ApJ, 562, 727

Izotov, Y. I., Papaderos, P., Guseva, N. G., Fricke, K. J., \& Thuan, T. X. 2004 A\&A, 421, 539

Kashikawa, N., Shimasaku, K., Malkan, M. A., et al. 2006, ApJ, 648, 7

Kennicutt, Jr., R. C. 1998, ARA\&A, 36, 189

Kodaira, K., Taniguchi, Y., Kashikawa, N., et al. 2003, PASJ, 55, L17

Kong, X., Charlot, S., Brinchmann, J., \& Fall, S. M. 2004, MNRAS, 349, 769

Kudritzki, R.-P., Méndez, R. H., Feldmeier, J. J., et al. 2000, ApJ, 536, 19

Kunth, D., Lequeux, J., Sargent, W. L. W., \& Viallefond, F. 1994, A\&A, 282, 709

Kunth, D., Mas-Hesse, J. M., Terlevich, E., et al. 1998, A\&A, 334, 11

Kunth, D., Leitherer, C., Mas-Hesse, J. M., Östlin, G., \& Petrosian, A. 2003, ApJ, 597, 263

Leitherer, C. 2005, in The Evolution of Starbursts, ed. S. Hüttmeister, E. Manthey, D. Bomans, \& K. Weis, AIP Conf. Ser., 783, 280
Leitherer, C., Schaerer, D., Goldader, J. D., et al. 1999, ApJS, 123, 3

Lequeux, J., Kunth, D., Mas-Hesse, J. M., \& Sargent, W. L. W. 1995, A\&A, 301, 18

Madau, P., Ferguson, H. C., Dickinson, M. E., et al. 1996, MNRAS, 283, 1388 Malhotra, S., \& Rhoads, J. E. 2004, ApJ, 617, L5

Margon, B., Anderson, S. F., Mateo, M., Fich, M., \& Massey, P. 1988, ApJ, 334, 597

Martins, L. P., Delgado, R. M. G., Leitherer, C., Cerviño, M., \& Hauschildt, P. 2005, MNRAS, 358, 49

Mas-Hesse, J. M., Kunth, D., Tenorio-Tagle, G., et al. 2003, ApJ, 598, 858

Meier, D. L., \& Terlevich, R. 1981, ApJ, 246, L109

Meurer, G. R., Heckman, T. M., \& Calzetti, D. 1999, ApJ, 521, 64

Moustakas, J., \& Kennicutt, Jr., R. C. 2006, ApJS, 164, 81

Murayama, T., Taniguchi, Y., Scoville, N. Z., et al. 2007, ApJS, 172, 523

Neufeld, D. A. 1991, ApJ, 370, L85

Oke, J. B. 1990, AJ, 99, 1621

Osterbrock, D. E. 1989, Astrophysics of gaseous nebulae and active galactic nuclei, Research supported by the University of California, John Simon Guggenheim Memorial Foundation, University of Minnesota, et al. (Mill Valley, CA, University Science Books), 422

Östlin, G., Zackrisson, E., Bergvall, N., \& Rönnback, J. 2003, A\&A, 408, 887

Östlin, G., Hayes, M., Kunth, D., et al. 2008, AJ, submitted

Ouchi, M., Shimasaku, K., Akiyama, M., et al. 2005, ApJ, 620, L1

Papaderos, P., Izotov, Y. I., Guseva, N. G., Thuan, T. X., \& Fricke, K. J. 2006, A\&A, 454, 119

Partridge, R. B., \& Peebles, P. J. E. 1967, ApJ, 147, 868

Pirzkal, N., Malhotra, S., Rhoads, J. E., \& Xu, C. 2007, ApJ, 667, 49

Pritchet, C. J. 1994, PASP, 106, 1052

Pritchet, C., \& Hartwick, D. 1989, JRASC, 83, 318

Rhoads, J. E., Dey, A., Malhotra, S., et al. 2003, AJ, 125, 1006

Salpeter, E. E. 1955, ApJ, 121, 161

Schaerer, D., \& Verhamme, A. 2008, A\&A, 480, 369

Schlegel, D. J., Finkbeiner, D. P., \& Davis, M. 1998, ApJ, 500, 525

Seibert, M., Martin, D. C., Heckman, T. M., et al. 2005, ApJ, 619, L55

Shapley, A. E., Steidel, C. C., Pettini, M., \& Adelberger, K. L. 2003, ApJ, 588, 65

Steidel, C. C., Giavalisco, M., Dickinson, M., \& Adelberger, K. L. 1996, AJ, 112,352

Taniguchi, Y., Ajiki, M., Nagao, T., et al. 2005, PASJ, 57, 165

Tapken, C., Appenzeller, I., Noll, S., et al. 2007, A\&A, 467, 63

Tenorio-Tagle, G., Silich, S. A., Kunth, D., Terlevich, E., \& Terlevich, R. 1999, MNRAS, 309, 332

Terlevich, E., Diaz, A. I., Terlevich, R., \& Vargas, M. L. G. 1993, MNRAS, 260, 3

Thuan, T. X., \& Izotov, Y. I. 1997, ApJ, 489, 623

Thuan, T. X., Izotov, Y. I., \& Lipovetsky, V. A. 1997, ApJ, 477, 661

Vázquez, G. A., \& Leitherer, C. 2005, ApJ, 621, 695

Verhamme, A., Schaerer, D., \& Maselli, A. 2006, A\&A, 460, 397

Verhamme, A., Schaerer, D., Atek, H., \& Tapken, C. 2007, A\&A, submitted 